NASA Technical Memorandum 84313

NASA-TM-8431319830009561

\title{
On the Structure of Wall-Bounded Turbulent Flows
}

John Kim

January 1983

LIBRARY GOPY

FFR? - 1983

LANGLEY RESEARCH CENTER

LIBRARY, NASA

HAMPTON, VIRGINIA 


\section{On the Structure of Wall-Bounded Turbulent Flows}

John Kim, Ames Research Center, Moffett Field, California 
Page intentionally left blank 
ABSTRACT

The variable-interval time-averaging (VITA) technique developed by Blackwelder and Kaplan [J. Fluid Mech. 76, 89 (1976)] is applied to data obtained from large-eddy simulation of turbulent channel flow in an investigation of the organized structures associated with the bursting phenomenon in the near-wall region. Conditionally averaged velocities, shcar stress, prcssurc, and vorticity are discussed in conjunction with the bursting phenomenon detected by the VITA technique. The conditionally averaged pressure reveals that the ejection process is associated with a localized adverse pressure gradient. In the plane perpendicular to the flow direction, the conditionally averaged vorticity field indicates that a pair of counterrotating streamwise vorticity is being lifted through the ejection process. 


\section{INTRODUCTION}

During the past decade, coherent structures in turbulent flow have been the subject of considerable interest among turbulence researchers. For a complete review of these structures, the reader should refer to the recent review article by Cantwell. ${ }^{1}$ In the present paper, the coherent structures associated with wall-bounded turbulent flows in the near-wall region are investigated. Substantial experimental evidence has been collected which indicates that there exist quasi-cyclic, spatially coherent structures in the vicinity of the wall in turbulent boundary layers. Although there is little disagreement about the existence of such organized structures, the lack of consensus on the detailed description of the structure still prevails (for example, see Kline and $\mathrm{Falco}^{2}$ ).

The bursting phenomenon is a subject of intense interest to researchers who are working to identify the organized structures in the wall-bounded shear flows. It is composed of a sequence of quasi-cyclic events that occur in the wall region. Extensive experimental work on the bursting phenomenon has been reported in the literature: e.g., Kline et a1., ${ }^{3}$ Corino and Brodkey, ${ }^{4}$ Brodkey et $a 1 .,{ }^{5}$ and 0 ffen and Kline. ${ }^{6}$ Blackwelder and Kaplan ${ }^{7}$ used a novel technique, called VITA (variable-interval time-averaging), to single out the bursting event from the usual chaotic turbulent flow. They then obtained the conditionally averaged statistics and reported some organized structures associated with the bursting phenomenon. In the present study, the same technique was applied to the data obtained from the large-eddy 
simulation of turbulent channel flow (Moin and $\mathrm{Kim}^{8}$ ), and the results are compared with the experimental results. In addition, the information on pressure and vorticity; which is not available from laboratory experiments, is analyzed in detail to gain a better understanding of the bursting phenomenon. In the aforementioned work of Moin and $\mathrm{Kim},{ }^{B}$ a fully developed turbulent channel flow was simulated numerically at a Reynolds number of 13,800 , based on the centerline velocity and channel half-width. The computed flow fleld was used to study the statistical properties of the flow as well as its time-dependent features. The agreement between the computed statistics and detailed flow structures and the experimental data was good. In addition, the turbulence structures in the vicinity of the wall of the channel flow were found to be the same as the structures in the wall region of turbulent boundary layers.

The terms "organized" and "coherent" structures have been used widely to imply different things by different investigators. It might be appropriate here to clarify their meaning in the present context to avoid a possible confusion. In the present paper, the words organized and coherent are used to imply structures that are recognizable through the ensemble-averaging process defined by the VITA technique. In other words, it is the structure that is ordered enough to be detected by the VITA technique.

In Sec. II the VITA technique is described briefly, and the results of the conditionally averaged fleld are presented in Sec. III. A short discussion and a conclusion based on the present investigation are 
presented in Sec. IV and a short summary of the present study is provided in Sec. $\mathrm{V}$.

\section{CONDITIONAL SAMPLING}

The detection of the bursting phenomenon can be very subjective depending on the investigator's detection criterion. Most of the information about the phenomenon is descriptive and is primarily based on flow-visualization experiments. If one tries to identify the event quantitatively, subjective criteria, such as the choice of detection function and threshold values, have to be introduced. Different investigators have used different techniques and have produced different results. In the present study, the VITA technique was used because it was simple and easy to implement, although not necessarily the best method available. The technique has been used previously by several investigators (Blackwelder and Kaplan, ${ }^{7}$ Blackwelder and Eckelmann, ${ }^{9}$ and Antonia et al., ${ }^{10}$ to name a few). The results of the present study can be compared with those of the previous ones. A brief description of the technique is given below, but the reader should refer to Ref. 7 for details.

The variable-interval time-average of a fluctuating quantity $Q\left(x_{1}, t\right)$ is defined as

$$
\hat{Q}\left(x_{i}, t, T\right)=\frac{1}{T} \int_{t-T / 2}^{t+T / 2} Q\left(x_{i}, \tau\right) d \tau,
$$

where $T$ is the averaging time. Note that

$$
\lim _{\mathrm{T} \rightarrow \infty} \hat{\mathrm{Q}}=\overline{\mathrm{Q}} \text {, }
$$


where the bar indicates the conventional time-average. If one wants to obtain a local average of some particular phenomenon, the averaging time $T$ must be of the order of the time-scale of the phenomenon under study. To represent a measure of the turbulent energy during time $T$, a localized variance is defined in the following way:

$$
\widehat{\operatorname{var}}\left(\mathrm{x}_{i}, t, T\right)=\widehat{u^{2}}\left(\mathrm{x}_{i}, t, T\right)-\left[\hat{u}\left(\mathrm{x}_{i}, t, T\right)\right]^{2},
$$

where $u$ is the fluctuating component of the streamwise velocity. The detection criterion for the bursting phenomenon is then completed by using a threshold level on the VITA variance. The detection function $D(t)$ is defined as

$$
D(t)= \begin{cases}1, & \text { for } \widehat{\operatorname{var}}>\mathrm{k} u_{\mathrm{rms}}^{2} \text { and } \partial u / \partial t>0, \\ 0, & \text { otherwise }\end{cases}
$$

where $k$ is the threshold level and $u_{r m s}$ is the root mean square of the fluctuating streamwise velocity. The second constraint, $\partial u / \partial t>0$, was not a part of the original detection function used by Blackwelder and Kaplan, ${ }^{7}$ but was added in the subsequent work of Chen and Balckwelder. ${ }^{11}$

The conditional average of a quantity $Q$ is defined by

$$
\left\langle Q\left(x_{i}, \tau\right)\right\rangle_{y^{+}}=\frac{1}{N} \sum_{j=1}^{N} Q\left(x_{i}, t_{j}+\tau\right),
$$

where $x_{i}$ is the position at which the sampling occurred, the subscript $\mathrm{y}^{+}$indicates the position at which detection occurred, and $\mathrm{N}$ is the total number of the events detected by Eq. (4).

To obtain a spatial structure rather than the temporal structure, in the present study, the conditional averaging process was modified 
in the following way to give the variable-interval space-averaging:

$$
\begin{aligned}
& \hat{Q}\left(x, y, z, t_{0}, L\right)=\frac{1}{L} \int_{x-L / 2}^{x+L / 2} Q\left(\xi, y, z, t_{0}\right) d \xi, \\
& \widehat{\operatorname{var}}=\widehat{u^{2}}-\hat{u}^{2}, \\
& D(x)= \begin{cases}1, & \text { for } \widehat{\operatorname{var}}>k u_{\mathrm{rms}}^{2} \text { and } \partial u / \partial x<0 \\
0, & \text { otherwise }\end{cases}
\end{aligned}
$$

and

$$
\left\langle Q\left(\xi, y, z, t_{0}\right)\right\rangle_{y^{+}}=\frac{1}{N} \sum_{j=1}^{N} Q\left(x_{j}+\xi, y, z, t_{0}\right),
$$

where $L$ is the width of the spatial averaging. (Note that most of the quantitative measurements produce temporal structures and that flow-visualization experiments generally yield spatial structures.) For all the data discussed in this paper, a threshold value of 1.2 and $L=8 \Delta x$, corresponding to 500 wall units (i.e., $L^{+} \equiv L u_{\tau} / \nu=500$ ), were used. The location of the detection probe was set at $y^{+}=21$. These values are comparable with the values used by the previous investigators. Blackwelder and Kaplan, ${ }^{7}$ for example, used $\mathrm{k}=1.2$ and $\mathrm{T}^{+} \equiv \mathrm{Tu}_{\tau}^{2} / \nu=10$ with the detection probe at $\mathrm{y}^{+}=15$. In addition, the ensembleaveraging process was taken over several flow fields at different time to obtain better statistics. Most of the results reported here were results of the ensemble averages over more than 1000 independent events.

\section{CONDITIONAL AVERAGES}

Figure 1 shows the conditionally averaged velocity profiles as functions of $\mathrm{y}^{+}$and the streamwise locations relative to the point of 
the detection. The location of the detection corresponds to $\xi=0$. The negative $\xi$ indicates upstream points of the detection, and the positive $\xi$ indicates downstream points of the detection. Each increment of $\xi$ corresponds to about 62 wall units (i.e., $\Delta \xi^{+}=62$ ). The dashed lines in the figure represent the mean-velocity profile and the symbols are the conditionally averaged profiles around the event. Recall that in contrast to the results of Blackwelder and Kaplan ${ }^{7}$ (BK, in short, hereinafter), the current results display the spatial structures of the event rather than the temporal structures. One can relate the present results to those of BK using the Taylor's hypothesis, in which case, the downstream separations correspond to the negative time delays and the upstream separation to the positive time delays; that is, one should scan Fig. 1 from the bottom right to the top 1eft. It appears the agreement between the two results is good. Both results show clearly the sweep-ejection process (ejection-sweep in the case of BK) through the detection point. These conditionally averaged profiles are shown as functions of $\mathrm{u}^{+}$and $\mathrm{y}^{+}$in Fig. 2. In Fig. 2(a), the timeaveraged wall-shear velocity $\overline{\mathrm{u}}_{\tau}$, was used to obtain the nondimensional variables $\mathrm{u}^{+}$and $\mathrm{y}^{+}$. With this nondimensionalization, the excess and the defect in the velocity during the event are confined within $\mathrm{y}^{+}$ between 10 and 70 . The outer parts of the profiles are not affected at al1. In Fig. 2(b), the conditionally averaged wall-shear velocity, $\left\langle u_{\tau}\right\rangle$, is used to nondimensionalize $u$ and $y$. With this nondimensionalization, the conditionally averaged velocity profiles follow closely the mean-velocity profile up to about $y^{+}=15$, suggesting that the 
velocity near the wall has adjusted rather quickly with the change in the wall shear. The deviation now is more pronounced in the outer part of the profiles.

Conditionally averaged profiles of $u, v, w$, and uv are shown in Fig. 3. The total length in the streamwise direction in the figure is about 4000 wall units. Other than the fact that one has to follow the figures from the right to the left, these figures are similar to those of BK. The only difference between the two results is that the magnitudes of the present results are consistently slightly lower than the experimental results. This could be attributed to the differences in parameters employed in the sampling process or, simply, to the difference between the temporal and spatial coherence. In any event, the difference does not seem to be significant. Note that in Fig. 3(a); the bottom curve represents the streamwise velocity at $\mathrm{y}^{+}=1.8$. Although it is not shown here, the conditionally averaged wall shear is very similar to this curve. One interesting feature of the curve is that it has a long tail to the upstream side (sweep side). This causes the deviation from the mean to become more noticeable further along the upstream side in Fig. 2(b).

The conditionally averaged pressure is shown in Fig. 4 together with the streamwise velocity. The pressure signatures show localized peaks associated with the event. As a result of these peaks, localized adverse pressure gradients are formed followed downstream by favorable pressure gradients around the detection point. Note that these peaks are more or less aligned in the direction normal to the wall. In contrast, the peaks of $u$ are skewed such that they arrive first away from 
the wall. The skewness of the u-signature can be interpreted as follows: (1) either the large-scale structure moves toward the wall at an oblique angle; or (2) the convection velocity of the large-scale structures is a function of the distance from the wall. The pressure profiles, however, indicate that the convection velocity of the pressure signature associated with the large-scale structure is constant across the layer. This produces a phase lag in the velocity signature relative to the pressure signature near the wall. From Fig. 4, it appears that the ejection process (the slowing-down of the streamwise velocity in the figure) is associated with the localized adverse pressure gradient near the wall. Based on this observation, the following statements can be made to describe the bursting event detected by the VITA technique: (1) the sweeping motion of the large-scale structure moves toward the wall at an oblique angle; (2) the pressure wave associated with the sweep has Iocalized peaks and precedes the velocity near the wall; (3) the fluid near the wall encounters the adverse pressure gradient and is ejected upward; and (4) as a result of this lift-up of the low-momentum fluid, the velocities away from the wall are decreased. Note that this description is consistent with the flow model of Offen and Kline, ${ }^{6}$ which describes the lift-up process as a response to a temporary local adverse pressure gradient.

The conditionally averaged spanwise vorticity is shown in Fig. 5. In the regions shown in the figure, most contributions to the vorticity derive from $-\partial u / \partial y$, and the contribution of $\partial v / \partial x$ is negligible. The profiles show the excess and defect vorticity associated with the sweep-ejection motions. The corresponding streamwise and normal 
vorticity were also computed; they showed no coherent motions in this $\xi$ - y plane, as expected by symmetry.

So far, we have examined the conditional averaged quantities in $\xi-y$ plane only. One can also study these profiles in $\xi-z$ and $y-z$ planes. In the $\xi-z$ plane, we can study the spanwise extent of the structure associated with the event. In Fig. 6, the conditionally averaged streamwise velocities, shear stresses, and pressure in this plane are shown at $y^{+}=21$ (note that this is the $y$-location of the detection point). The spanwise correlations of $u$ and $u v$ vanish beyond $z^{+} \cong \pm 30$, whereas those of the pressure seem to extend to about \pm 60 . (The scaling of the large-scale structure was not captured properly in the simulation of Moin and $\mathrm{Kim}^{8}$ because of the numerical resoIution problem; therefore, any quantitative information must be taken with some care.) This larger spanwise extent of the pressure eddies was also apparent from the contour plots of the instantaneous pressure (see Moin and $\mathrm{Kim}^{8}$ ). The conditionally averaged spanwise velocity profiles are shown in Fig. 7. The direction is such that the positive $w$ represents moving downward (i.e., to the positive $z$ ), and the negative corresponds to moving upward (i.e., to the negative $z$ ). The profiles indicate the spanwise outward motions during the sweep and the inward motions during the ejection. These spanwise outward motions during the sweep, referred to as "splatting" by Moin and Kim, ${ }^{8}$ resulted in a peculiar intercomponent energy transfer in the wall region.

The conditionally averaged streamwise vorticity in $y-z$ planes is shown in Fig. 8 at various streamwise locations. Note that flow goes into the plane in these figures; we then can investigate the 
streamwise vorticity which is associated with the sweep-ejection process. The total spanwise width is about 2000 wall units. The sense of rotation is such that positive vorticity indicates clockwise rotation and negative vorticity indicates counterclockwise rotation. Figure $8(a)$ shows the profiles of the vorticity at $\xi=-10$, which is about 620 wall units upstream of the detection point (recall that $\xi=0$ is at the center of the event and each increment corresponds to 62 wall units). At this upstream location, no detectable correlation is found. Figure $8(b)$ is the vorticity signatures at $\xi=-7$, and we begin to see that an appreciable amount of the streamwise vorticity components survived the ensemble-averaging process.

Figures $8(\mathrm{c})$ through $8(\mathrm{k})$ show how the streamwise vorticity signatures change their patterns as we move downstream through the center of the event. Several points need to be addressed explicitly here. First, the streamwise vorticity appears as a counterrotating pair, and there seems to be two distinct groups: one near the wall and the other slightly away from the wall. These two groups have opposite signs of vorticity as a pair. Whether this streamwise vorticity is associated with the actual revolving vortex motion cannot be determined from the current figures. However, in the previous work of Moin and $\mathrm{Kim}^{8}{ }^{8}$ it was found that the streamwise vorticity very close to the wall, say $\mathrm{y}^{+}$less than 10, was due to the gradient of spanwise velocity, whereas some of the vorticity at $\mathrm{y}^{+}$between 20 to 100 was indeed associated with the revolving vortex motion. Next, the magnitudes of the vorticity near the wall increase as we approach the detection point, and the vorticity that existed away from the wall slowly disappears; in fact at 
$\xi=0$, only the former group is noticeable. Also we note that the center of the pair of the vorticity (the $y$ location of the maximum strength) of the former group starts at the wall at $\xi=-5$ and moves upward through the detection point $\left(\mathrm{y}^{+}=21\right.$ is the center at this point) and moves further upward $\left(y^{+}=46\right.$ at $\left.\xi=2\right)$ before it loses its identity far downstream. These figures suggest that the pair of counterrotating streamwise vorticity which is generated near the wal1 Is being lifted-up through the ejection process. It is possible that this pair of counterrotating vorticity is in fact the two legs of the horseshoe vortex referred to by offen and Kline ${ }^{6}$ in their flow model of the near-wall region, or the hairpin-type vortices observed farther out in the boundary layer by Head and Bandyopadhyay. ${ }^{12}$ The angle of the lifting vorticity with respect to the wall is roughly estimated to be about $10^{\circ}$ at the center of the event, where the center of the vorticity is at $y^{+}=21$. This angle is far from the $45^{\circ}$ of the hairpin vortices measured by Head and Bandyopadhyay. ${ }^{12}$ However, recall that we are still very close to the wall and that this angle is in agreement with the angle of large-eddy structures close to the wall reported by Rajagopalan and Antonia. ${ }^{13}$

Finally, one more point needs to be mentioned in conjunction with the figures. Downstream of the center of the event, a new pair of counterrotating vorticity appears, with the opposite sign of rotation relative to the pair of vorticity being lifted. This vorticity is the result of the upward motion and the no-slip boundary condition. 
IV. DISCUSSION

Lately some investigators have suggested that a turbulent boundary layer is composed almost exclusively of hairpin or horseshoe vortices. However, this may be too simplified a picture of the complicated turbulent flow. There exists some evidence that the hairpin and horshoe vortices do exist in the turbulent boundary layer, but they may not be the only structures that exist in the flow. Moin ${ }^{14}$ recently examined carefully the data of Moin and $\mathrm{Kim}^{8}$ and also found some evidence of the hairpin-type vortices. However, he also found many other structures in addition to the motion of the hairpin-type vortices. The present study seems to suggest that the pair of counterrotating vorticity is a dominant structure during the bursting event (in the sense that they survive the ensemble-averaging process). Nevertheless, the possibility of whether this streamwise vorticity is indeed a part of the two legs of the hairpin-type vortices is still an open question.

Based on the results presented in the previous section, one can make the following conclusions with regard to the bursting event detected by the VITA technique (a simple sketch characterizing this flow pattern is shown in Fig. 9):

1. High-speed fluid moves toward the wall at an oblique angle during the sweep. Unfortunately the cause of this sweeping motion is not known at present.

2. The impingement of the high-speed fluid at the wall (splatting) and the viscous boundary condition create a pair of counterrotating, streamwise vorticity. 
3. As this vortical fluid moves downstream, it encounters a localized adverse pressure gradient, which was set up by the sweeping motion (the pressure wave moves ahead of the sweeping fluid), and is ejected upward.

4. As this pair of the streamwise vorticity is being lifted, they become a pair of counterrotating streamwise vortices (this notion of vortices rather than the vorticity, however, is inferred from the previous study of Moin and $\mathrm{Kim}^{8}$ ).

5. This pair of the counterrotating vortices scoops up more lowspeed fluid in between the vortices, and the flow in the large portion of the inner layer decelerates. They also create a pair of streamwise vorticity of opposite signs with respect to them near the wall because of the viscous boundary condition.

Note that this description of the bursting event is generally consistent with the flow model proposed by Blackwelder and Eckelmann ${ }^{9}$ on the role of the streamwise vortices for the event. However, the streamwise extent of these vortices is shorter than the one their model suggests. It appears that these streamwise vortices are much shorter than the wall layer streaks, suggesting that the streamwise vortices are needed only for initiating the formation of the streaks and that they do not have to be present side by side with the streaks. In addition, these streamwise vortices are tilted with respect to the wall. This is consistent with the result of Kim and Moin, ${ }^{15}$ which showed that the contour plots of the streamwise vorticity did not exhibit any streaky structure in contrast to the contours of the streamwise velocity. 


\section{SUMMARY}

This paper reports an attempt to identify some organized structures of wall-bounded shear flows using the results obtained from a threedimensional time-dependent numerical simulation. The availability of the three-dimensional turbulent flow field allowed the author to investigate the spatial structures associated with the bursting phenomenon. The VITA technique developed by Blackwelder and Kaplan ${ }^{7}$ was applied to the flow field to detect the bursting events. The present results agree well with available experimental data, if one converts the temporal structures of the experimental data to the spatial structure, using the Taylor's hypothesis. Upon this validation, the present study was extended to include conditionally averaged pressure and vorticity. The conditionally averaged pressure revealed that the ejection process at the end of the sweeping motion was associated with the localized adverse pressure gradient. This result was consistent with the flow model of Offen and Kline, ${ }^{6}$ although their model was based on a speculation because of the lack of pressure data associated with their visual data. The current results confirmed their hypothesis that the lift-up of the inner layer may be akin to convected separation brought about by a temporary local adverse pressure gradient.

The conditionally averaged profiles of streamwise vorticity in the y - z plane indicated that a pair of counterrotating vorticity was being lifted up through the ejection process. This streamwise vorticity was initially formed at the wall as the result of "splatting" and the viscous boundary condition. Although the present study indicated that 
the pair of counterrotating streamwise vorticity is a dominant structure for the bursting event, further work is required to determine whether this structure is related to the horseshoe vortex of offen and Kline ${ }^{6}$ or to the hairpin vortices of Head and Bandyopadhyay. ${ }^{12}$ 
ACKNOWLEDGMENT

4

I am grateful to $P$. Moin for numerous helpful discussions throughout the course of this work; to A. Leonard for his useful comments on the material presented in this paper; and to Professor R. F. Blackwelder, University of Southern California, for providing the author with the information on the VITA technique. 
REFERENCES

${ }^{1}$ B. J. Cantwell, Ann. Rev. F1uid Mech. 13, 457 (1981).

${ }^{2}$ S. J. Kline and R. E. Falco, Summary of the AFOSR/MSU Research Specialists Workshop on Coherent Structure in Turbulent Boundary Layers, AFOSR TR-80-0290, Dept. of Mechanical Engineering, Michigan State University (1980).

${ }^{3}$ S. J. Kline, W. C. Reynolds, and F. A. Schraub, J. Fluid Mech. 30, 741 (1967).

${ }^{4}$ E. R. Corino and R. S. Brodkey, J. Fluid Mech. 37, 1 (1978).

${ }^{5}$ R. S. Brodkey, J. M. Wallace, and H. Eckelmann, J. Fluid Mech. 63, 209 (1974).

${ }^{6}$ G. R. Offen and S. J. Kline, J. Fluid Mech. 70, 209 (1975).

${ }^{7}$ R. F. Blackwelder and R. E. Kaplan, J. Fluid Mech. 76, 89 (1976).

${ }^{8}$ P. Moin and J. Kim, J. Fluid Mech. 118, 341 (1982).

${ }^{9}$ R. F. Blackwelder and H. Eckelmann, J. Fluid Mech. 94, 577 (1979).

${ }^{10}$ R. A. Antonia, S. Rajagopalan, C. S. Subramanian, and A. J. Chambers, J. Fluid Mech. 121, 123 (1982).

${ }^{11}$ C. P. Chen and R. F. Blackwelder, J. Fluid Mech. 89, 577 (1978).

${ }^{12}$ M. R. Head and P. Bandyopadhyay, J. Fluid Mech. 107, 297 (1981).

${ }^{13}$ S. Rajagopalan and R. A. Antonia, Phys. Fluids 22, 614 (1979).

${ }^{14} \mathrm{P}$. Moin (private communication).

${ }^{15} \mathrm{~J}$. Kim and P. Moin, Proceedings of AGARD Conference on Turbulent Boundary Layers - Experiments, Theory and Modeling, AGARD CP No. 271, 1979. 


\section{FIGURE CAPTIONS}

FIG. 1. Conditionally averaged and mean-velocity profiles with upstream and downstream separation relative to the point of detection. $\left(000=\langle\mathrm{u}\rangle / \mathrm{U}_{\infty} ;-\cdots, \overline{\mathrm{u}} / \mathrm{U}_{\infty}\right)$.

FIG. 2. Conditionally averaged and mean-velocity profiles as functions of $\mathrm{y}^{+}$and $\mathrm{u}^{+}$: (a) $\mathrm{y}^{+}$and $\mathrm{u}^{+}$are nondimensionalized by the mean-wa11shear velocity; (b) $\mathrm{y}^{+}$and $\mathrm{u}^{+}$are nondimensionalized by the conditionally averaged wall-shear velocity $\left(000=\langle\mathrm{u}\rangle / \mathrm{u}_{\tau} ;--,=\overline{\mathrm{u}} / \mathrm{u}_{\tau}\right)$.

FIG. 3. The conditional averages in $\xi-y$ plane. The detection was applied at $\mathrm{y}^{+}=21$ : (a) streamwise velocities; (b) normal velocities; (c) spanwise velocities; (d) Reynolds shear stress.

FIG. 4. The conditional averages of the streamwise velocity and pressure $(-=\langle u\rangle ;--\langle p\rangle)$.

FIG. 5. The conditional averages of the spanwise vorticity.

FIG. 6. The conditional averages as a function of the spanwise coordinate at $y^{+}=21$ : (a) streamwise velocity; (b) Reynolds shear stress; (c) pressure.

FIG. 7. Conditionally averaged spanwise velocities as a function of the spanwise coordinate at $y^{+}=21$.

FIG. 8. Conditionally averaged streamwise vorticity as functions of the normal and spanwise coordinates: (a) $\xi=-10$; (b) $\xi=-7$; (c) $\xi=-5$;
(d) $\xi=-3$;
(e) $\xi=-2$;
(f) $\xi=-1$;
(g) $\xi=0$;
(h) $\xi=1$;
(1) $\xi=2$;
(j) $\xi=3$; and (k) $\xi=5$. 
FIG. 9. Flow patterns and associated vorticity during the sweep and ejection process: (a) end view; (b) side view. 

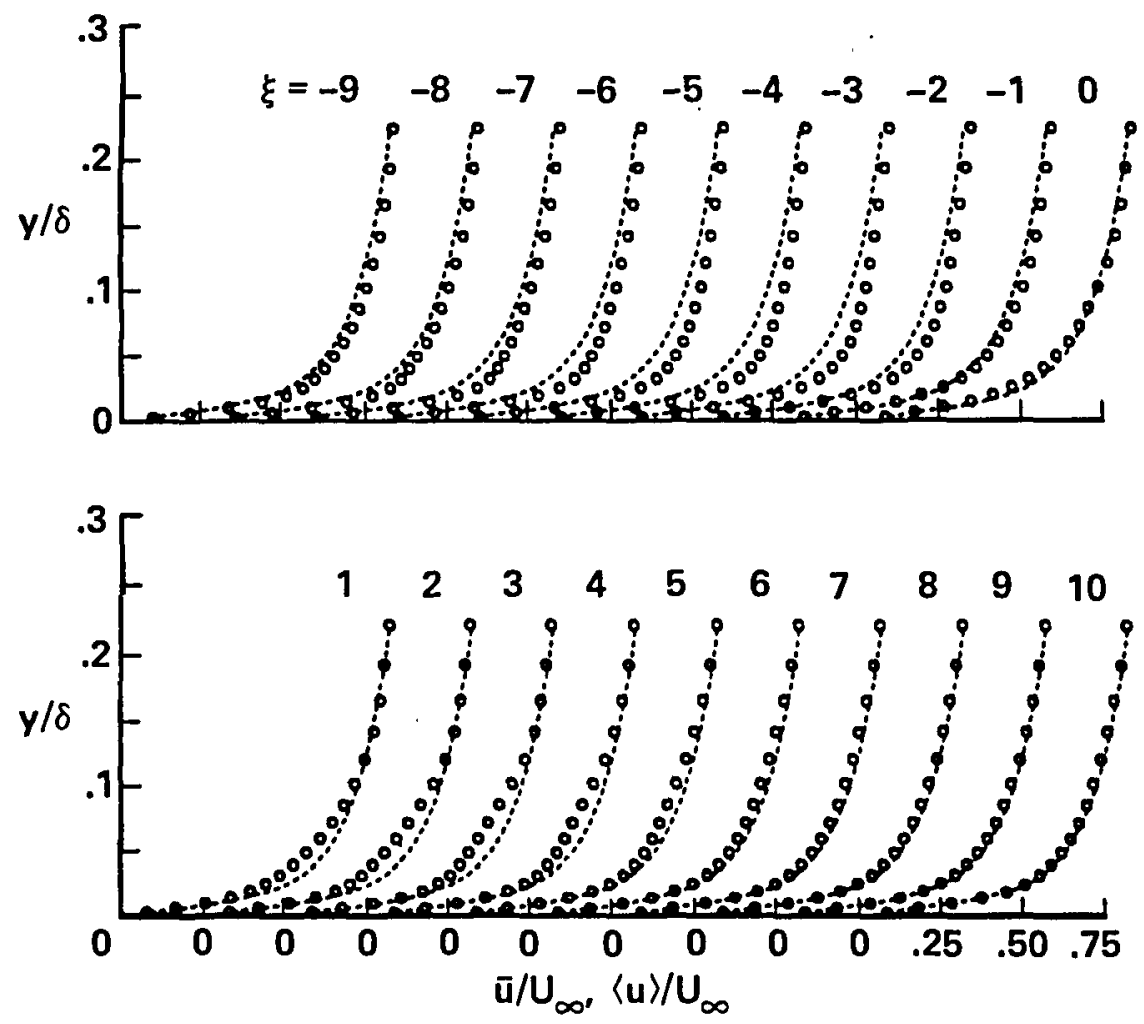

Fig. 1 


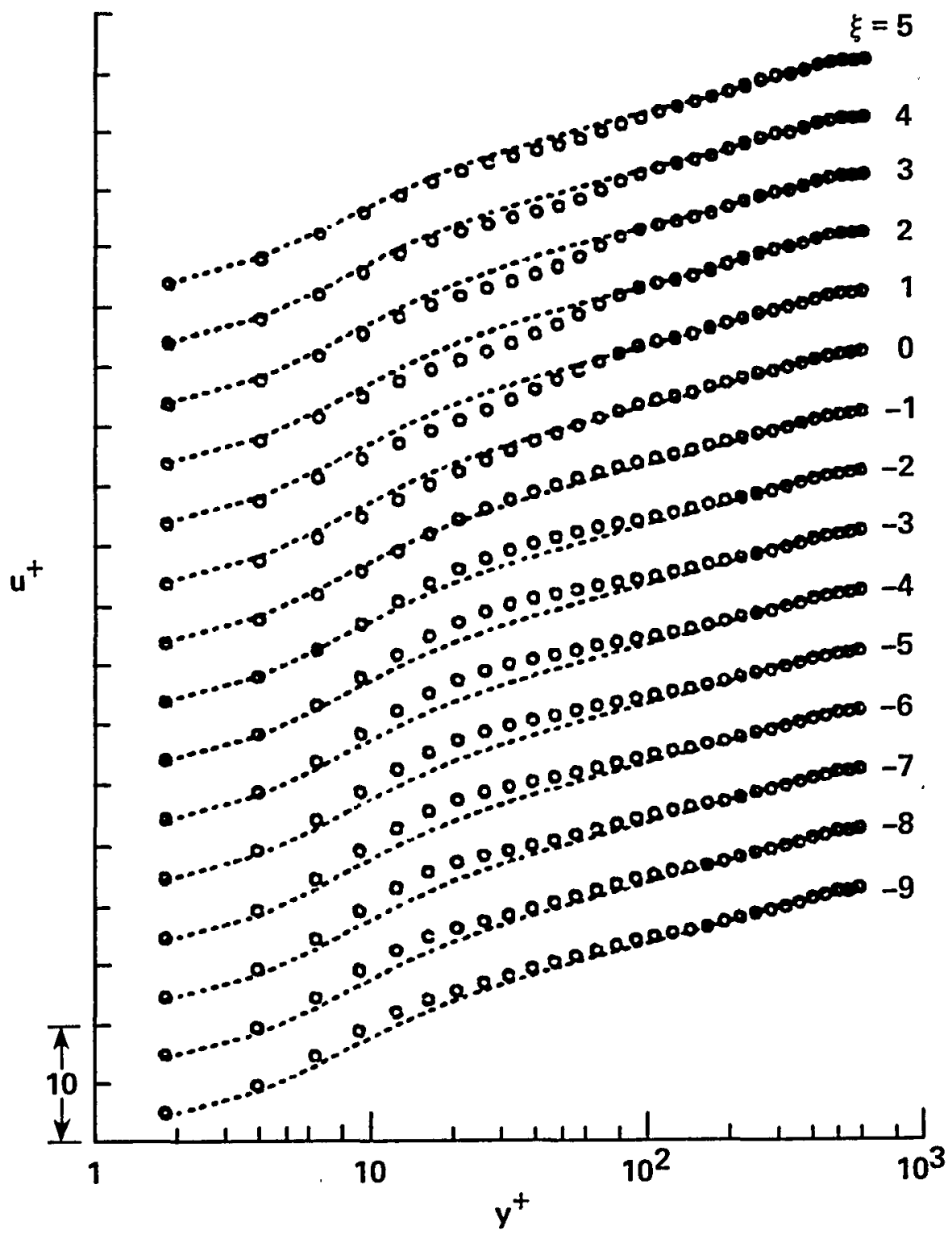

Fig. 2 a 


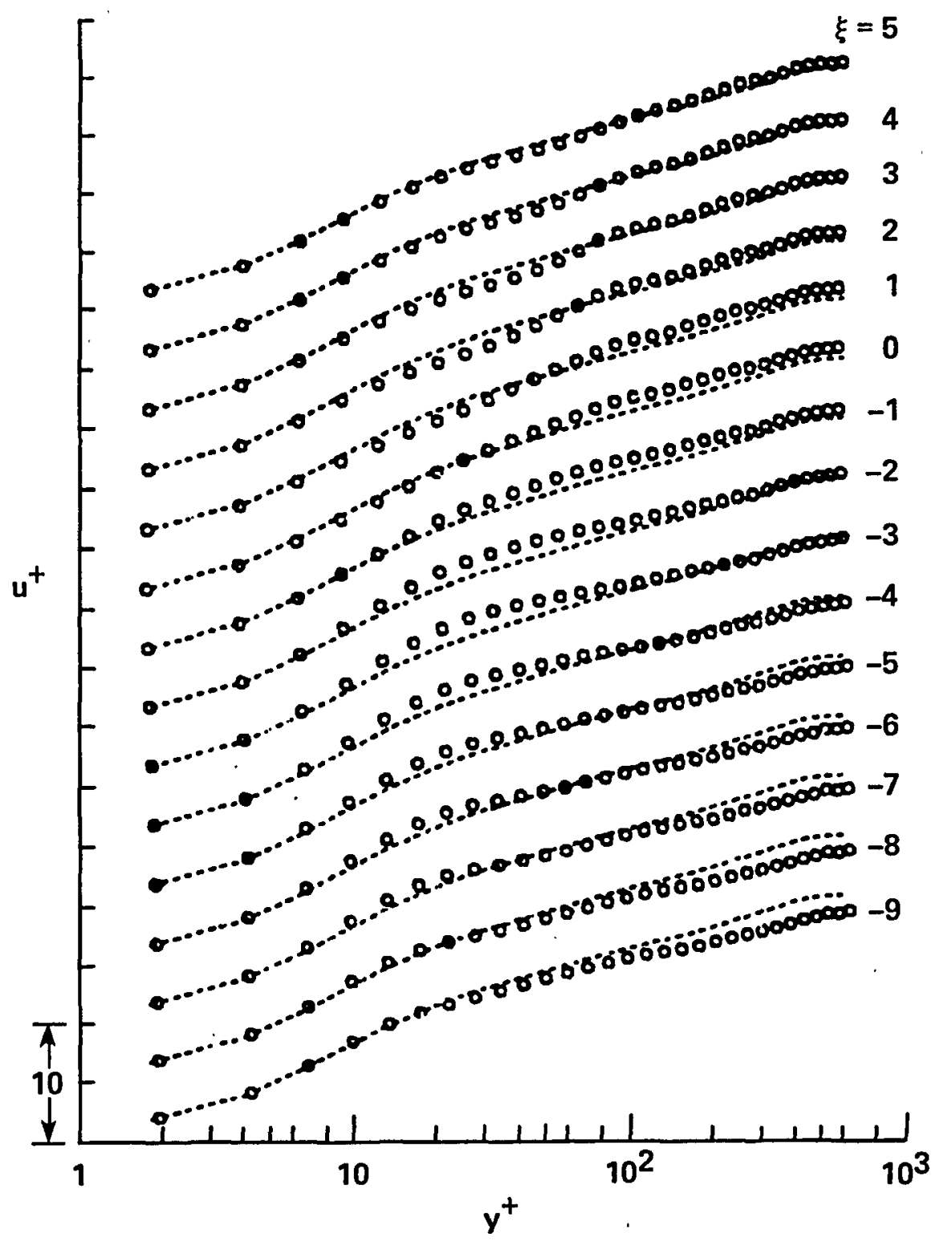

Fig. 2b 


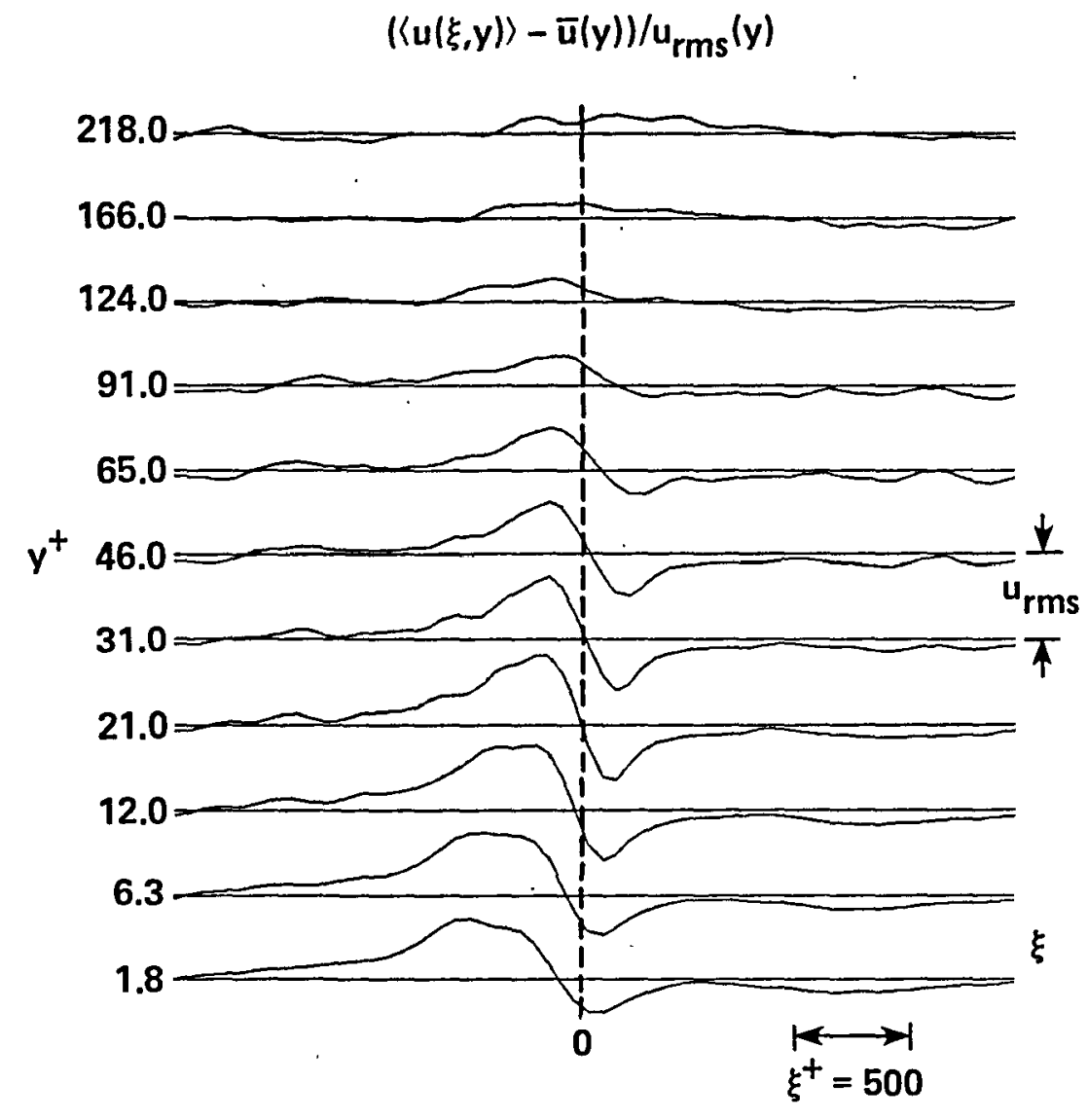

Fig. 3a 


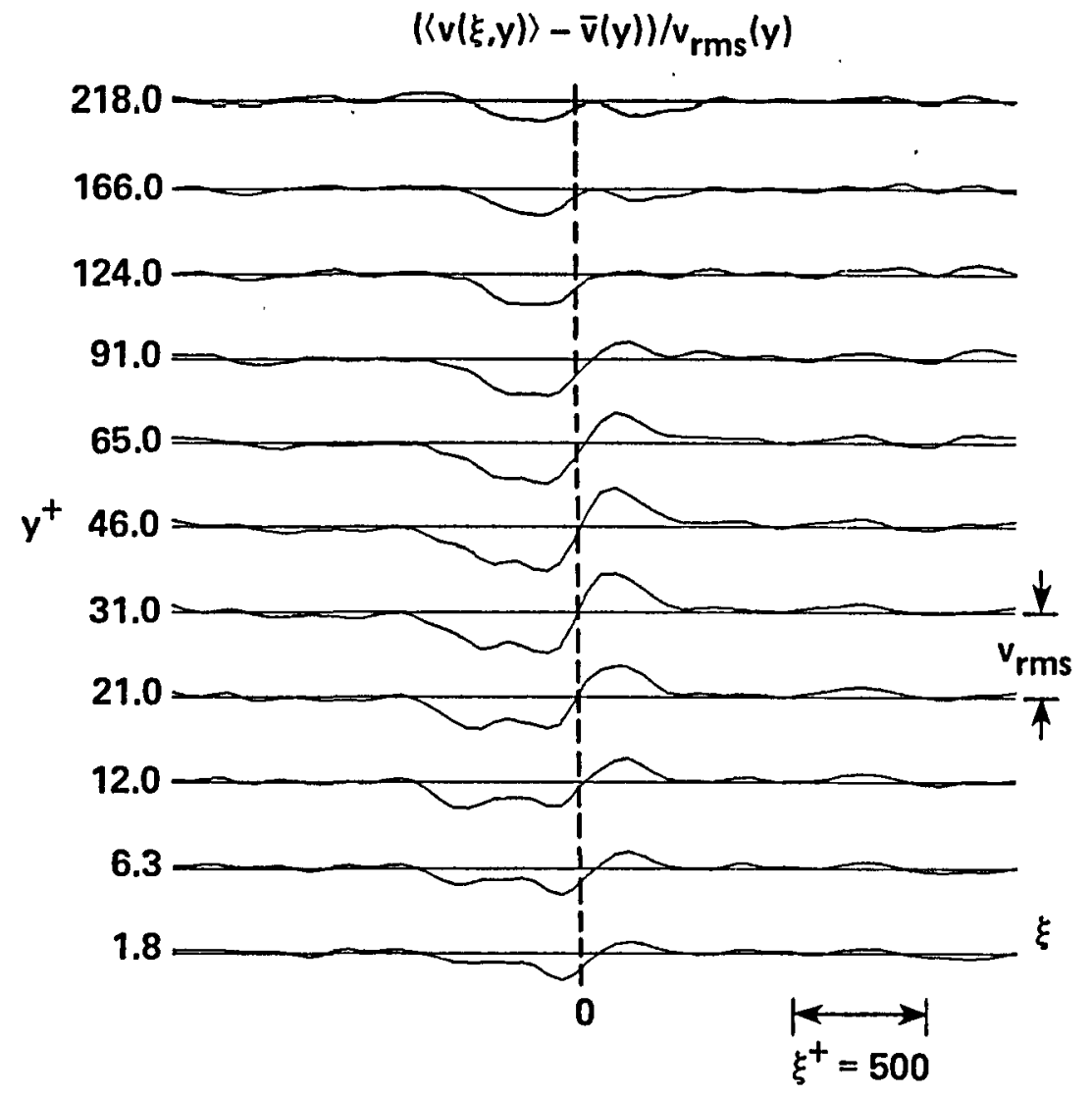

Fig. $3 b$ 


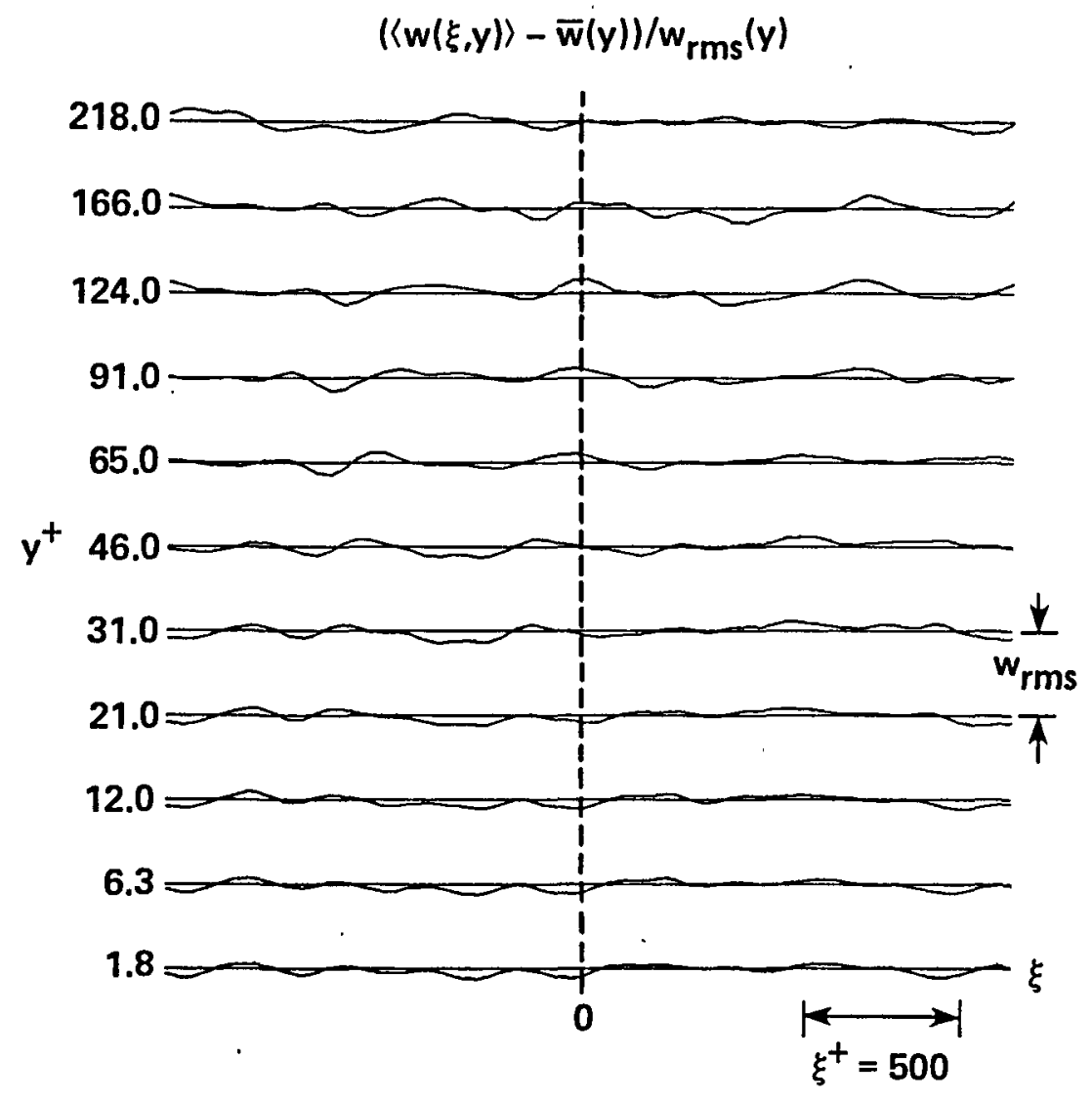

Fig. $3 c$ 


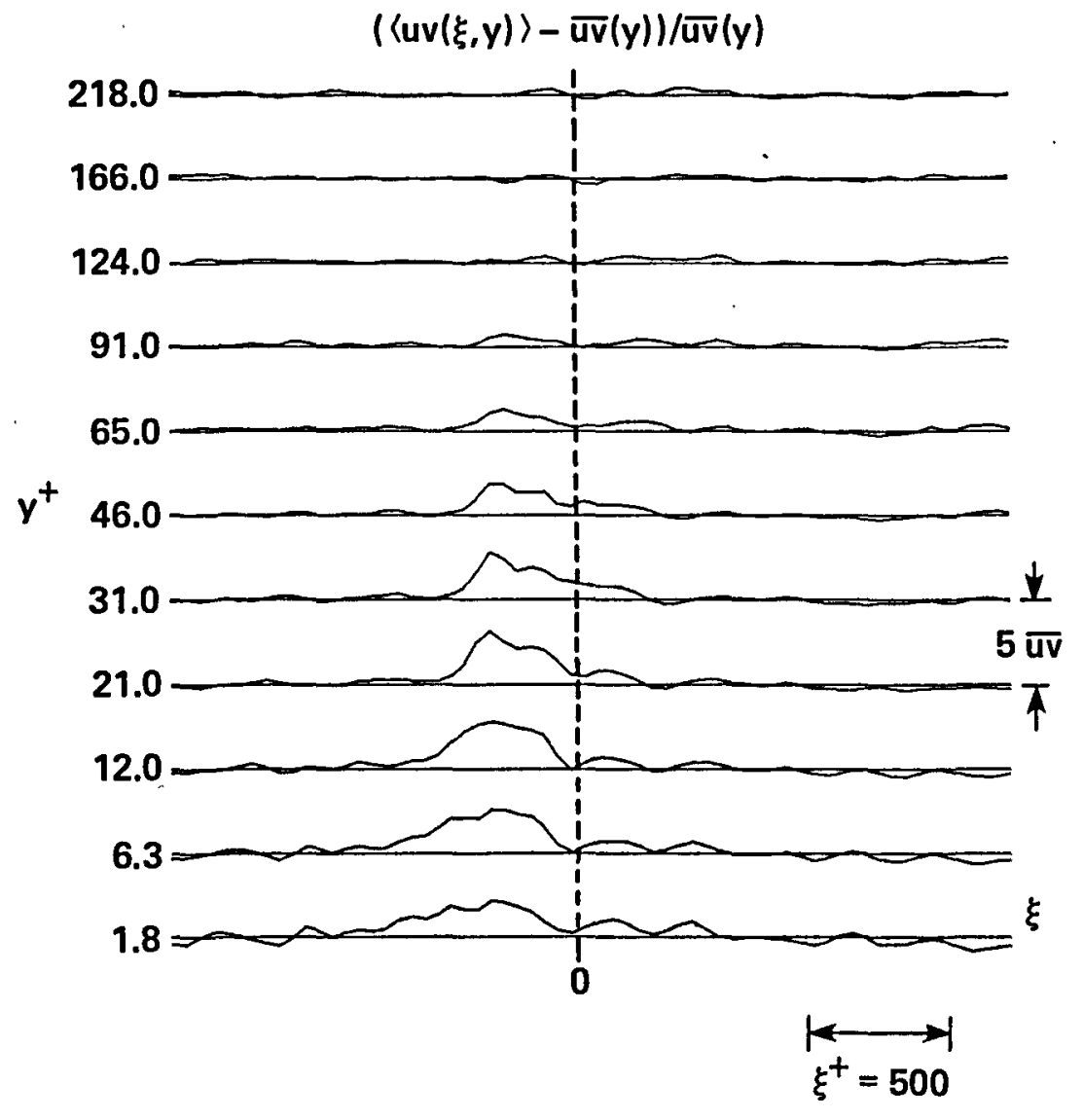

Fig. 3d 


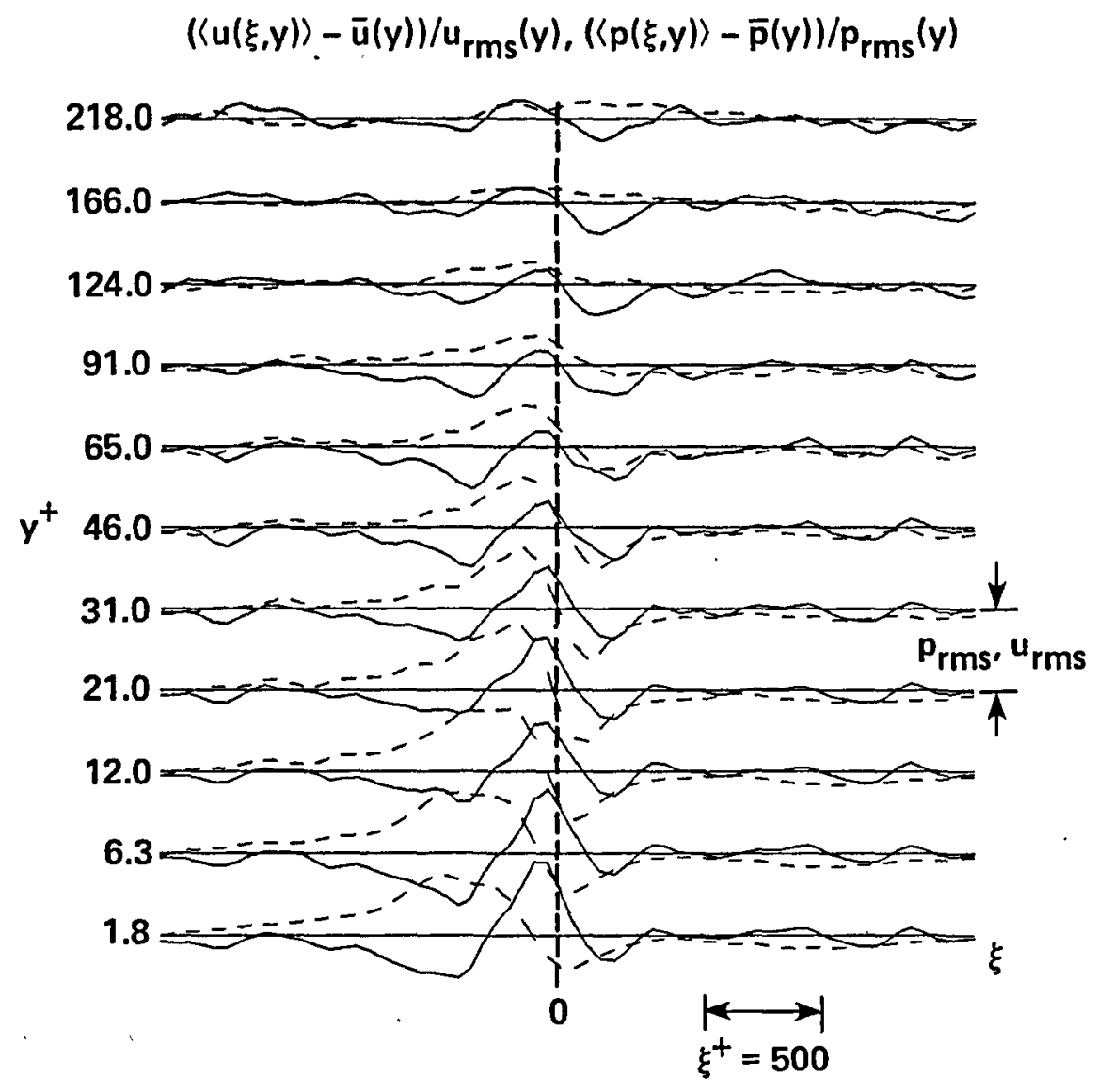

Fig. 4 


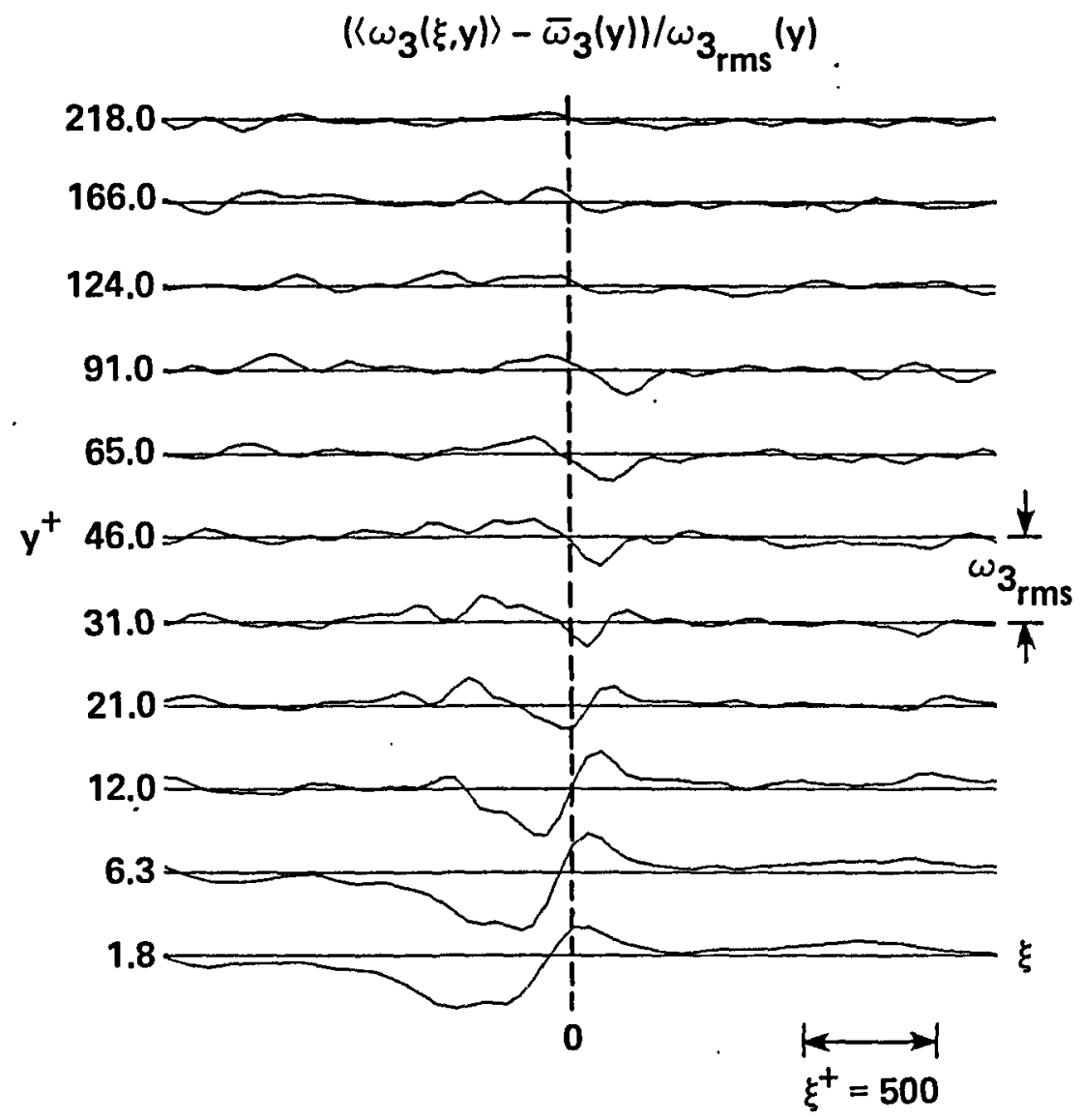

Fig. 5 


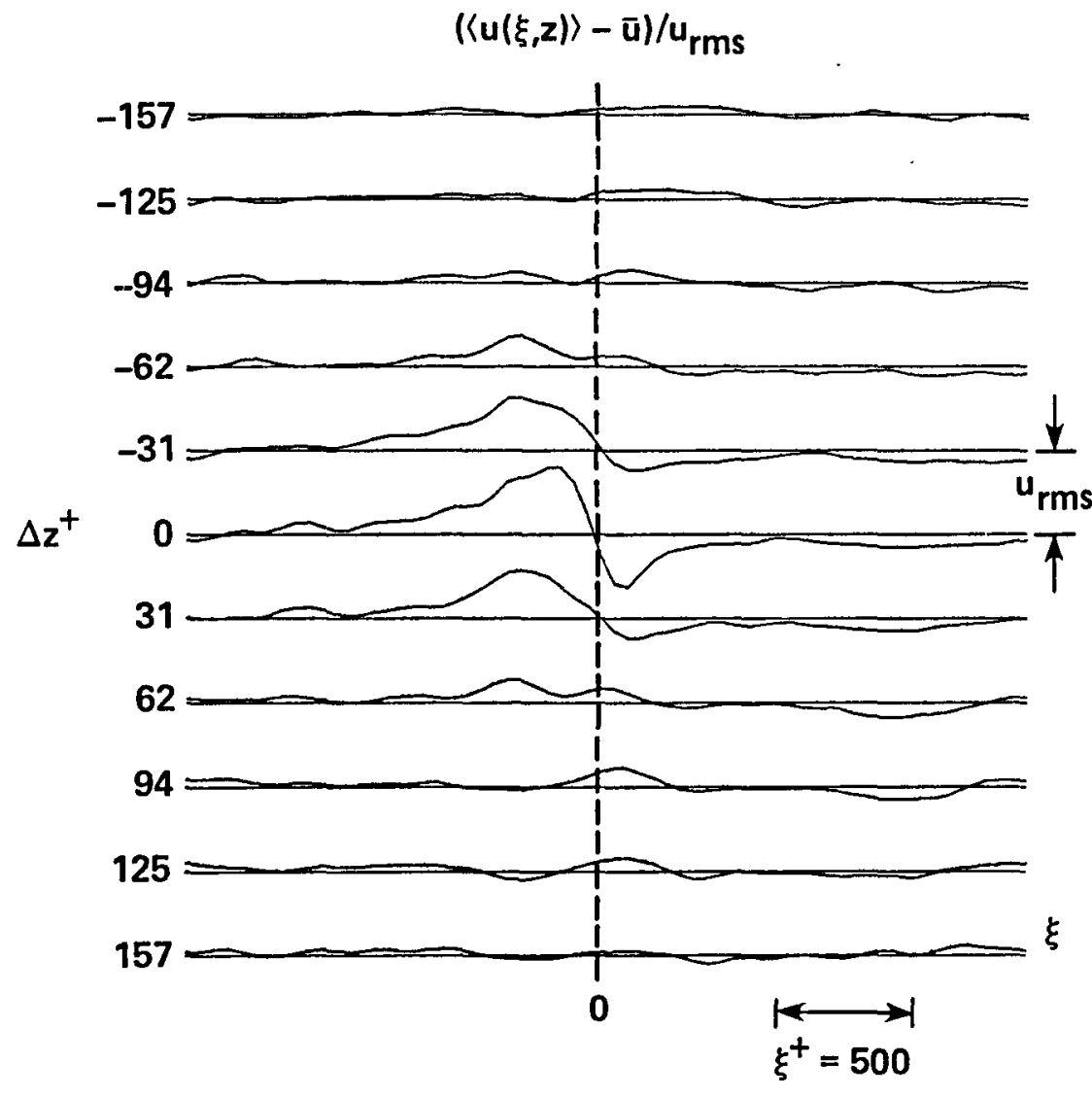

Fig. $6 a$ 


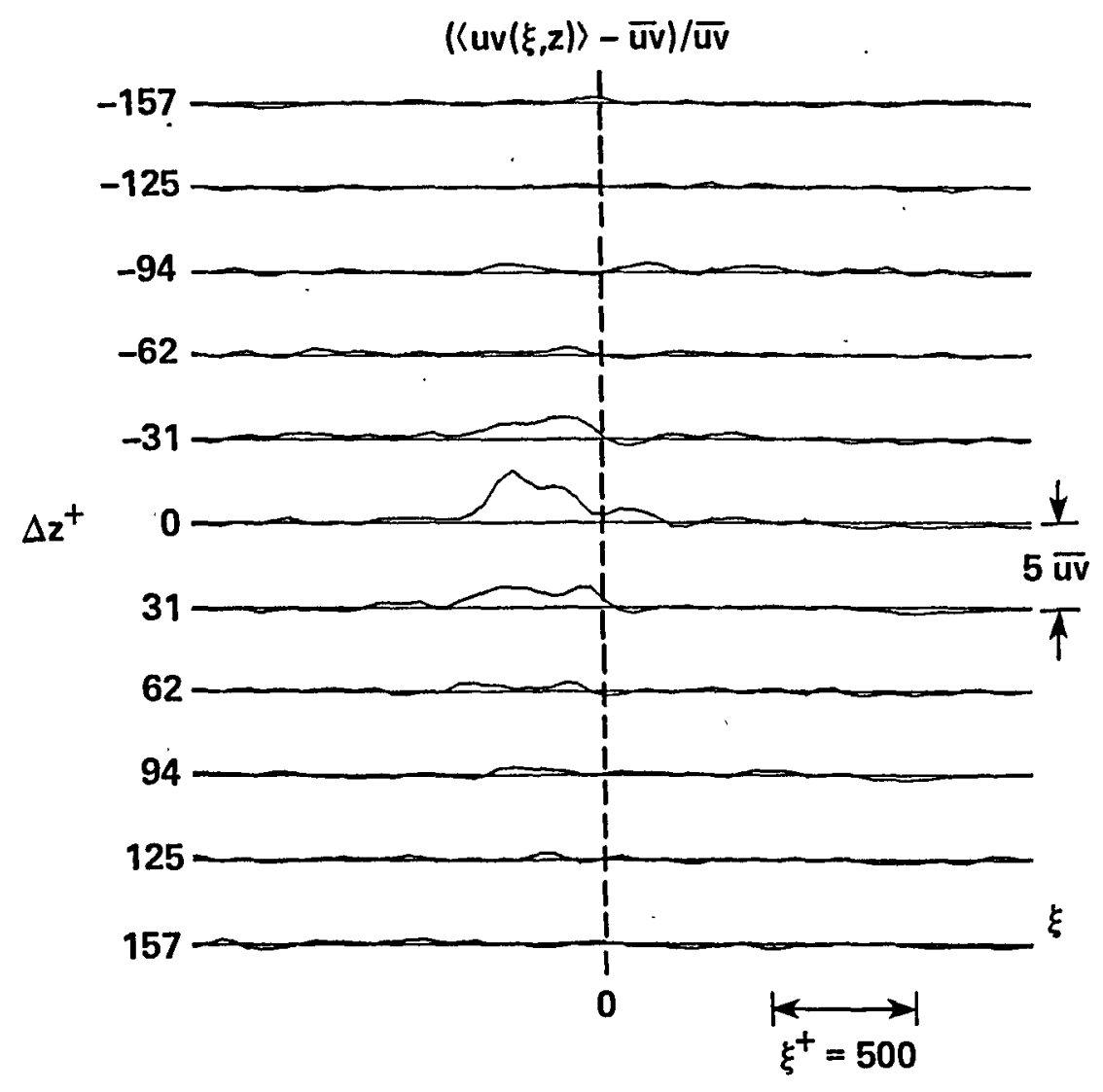

Fig. 6b 


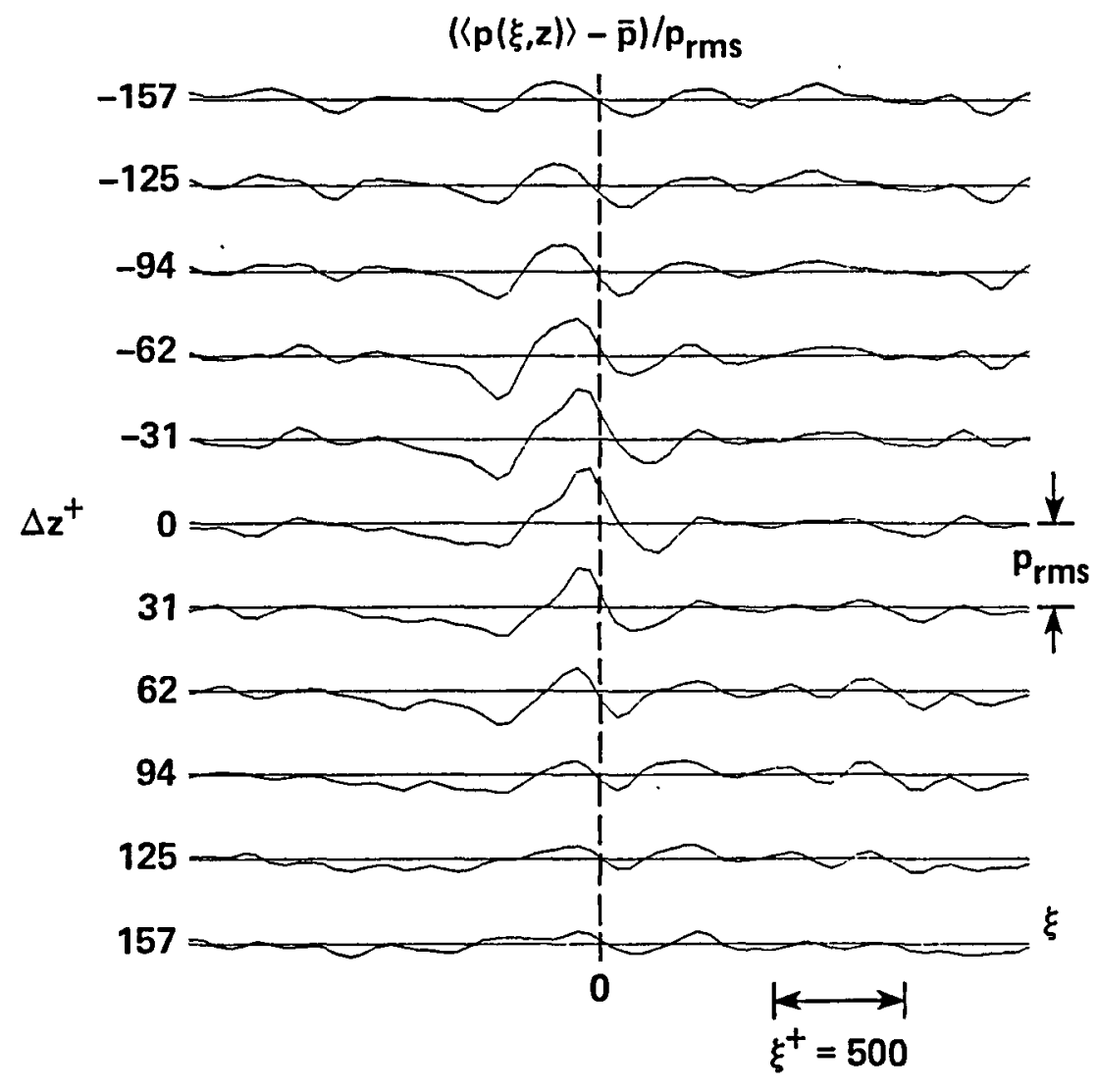

Fig. 6c 


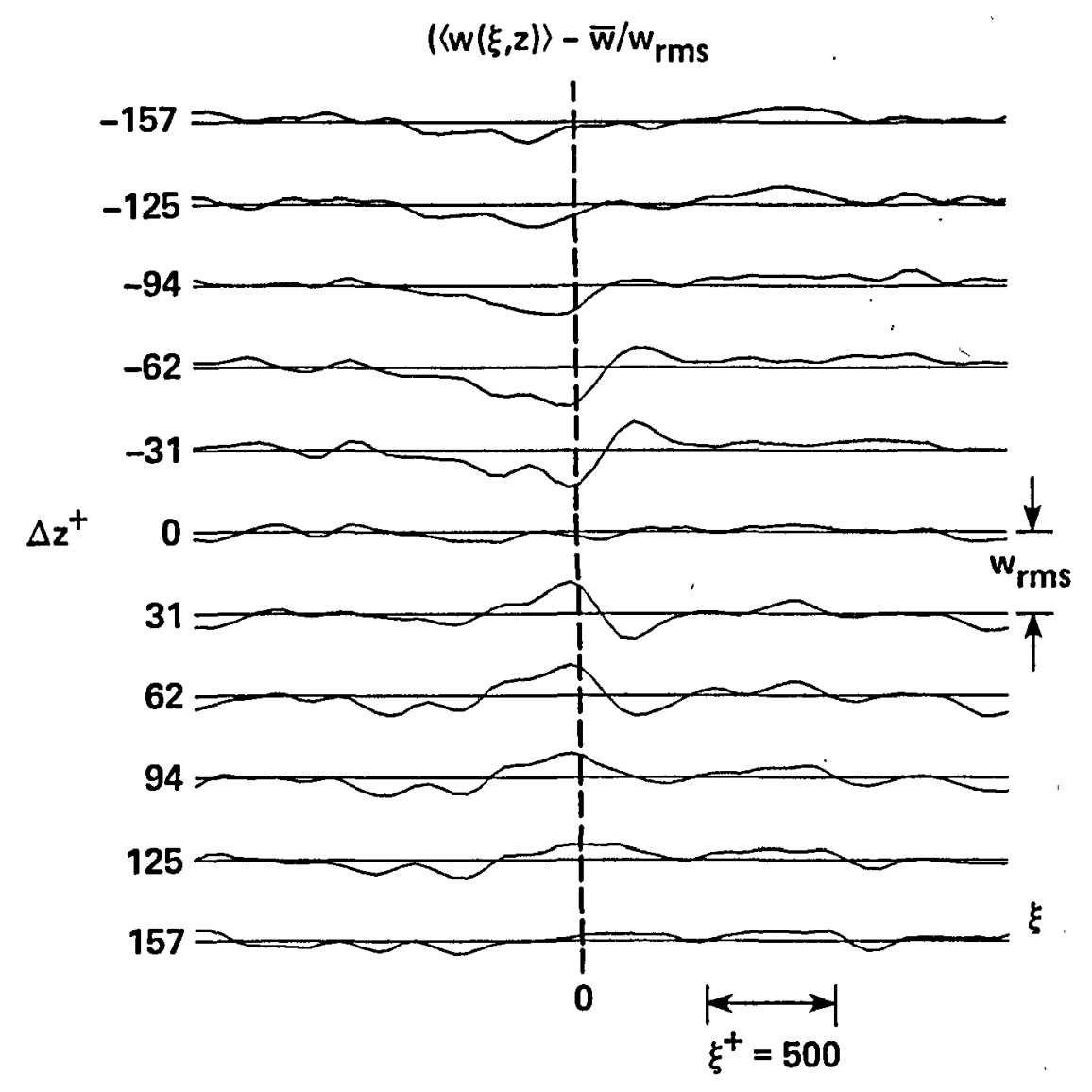

Fig. 7 


$$
\left\langle\left\langle\omega_{1}(y, z)\right\rangle-\bar{\omega}_{1}(y)\right) / \omega_{1_{\mathrm{rms}}}(y)
$$

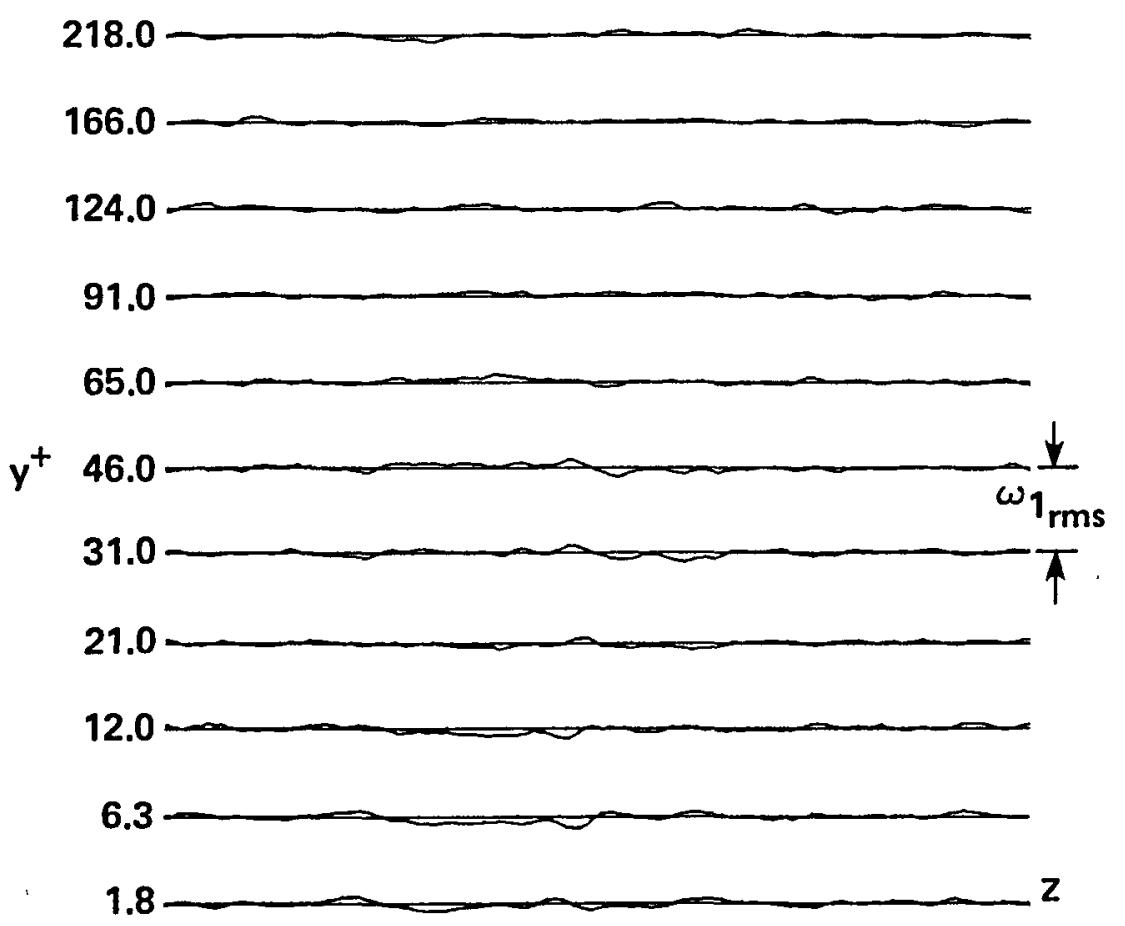

Fig. 8a 
$\left\langle\left(\omega_{1}(y, z)\right\rangle-\omega_{1}(y)\right) / \omega_{1_{r m s}}(y)$

218.0

166.0

$124.0=$

91.0

65.0

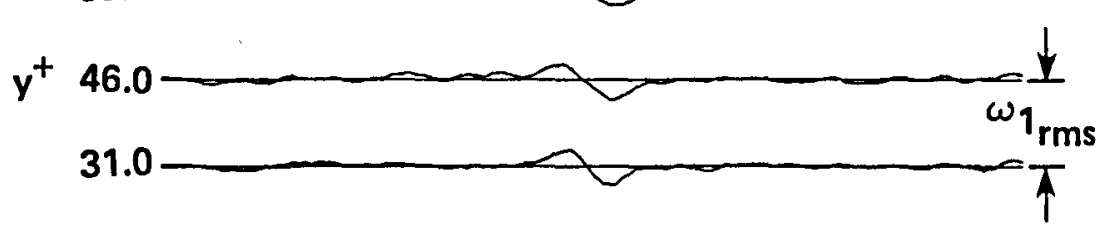

21.0

12.0

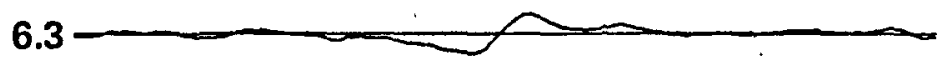

$1.8 \longrightarrow 2$

Fig. $8 \mathrm{~b}$ 


$$
\left(\left\langle\omega_{1}(y, z)\right\rangle-\bar{\omega}_{1}(y)\right) / \omega_{1_{\mathrm{rms}}}(y)
$$

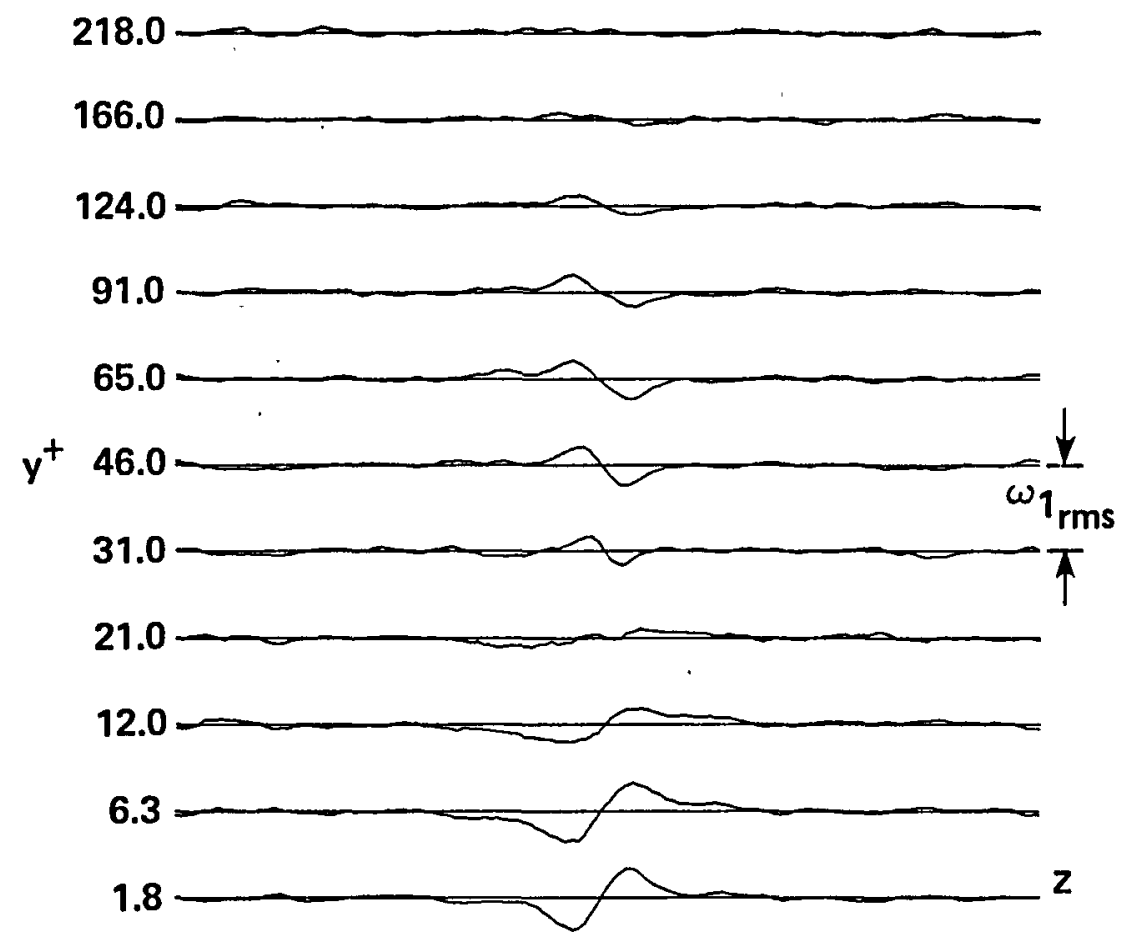

Fig. $8 c$ 


$$
\left(\left\langle\omega_{1}(y, z)\right\rangle-\bar{\omega}_{1}(y)\right) / \omega_{1_{\mathrm{rms}}}(y)
$$

$$
218.0
$$

166.0
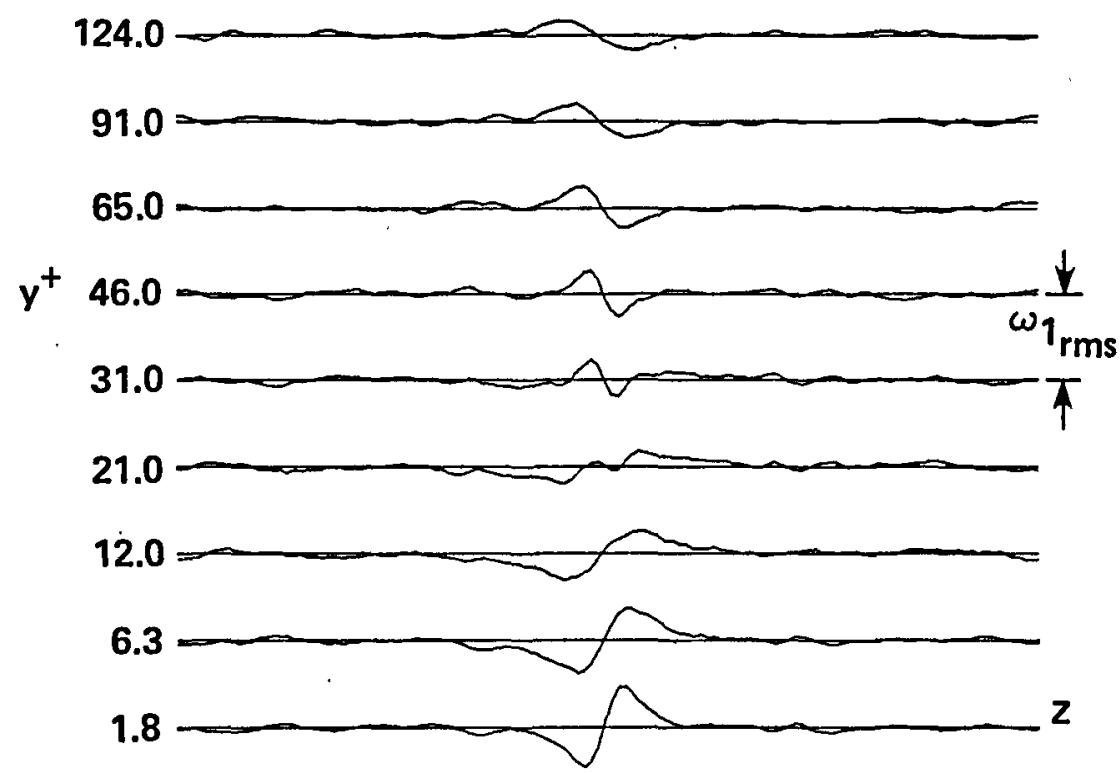

Fig. 8d 


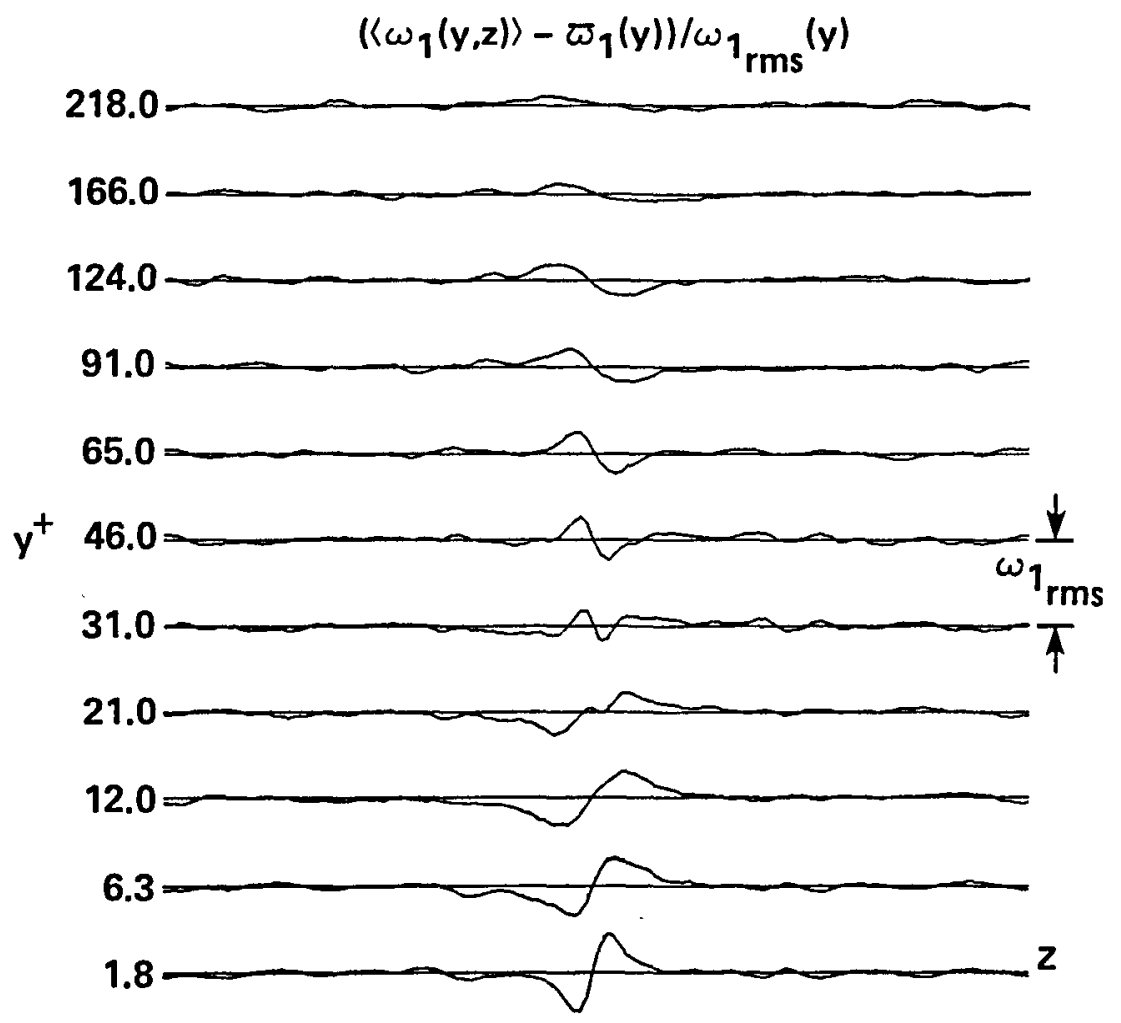

Fig. $8 \mathrm{e}$ 


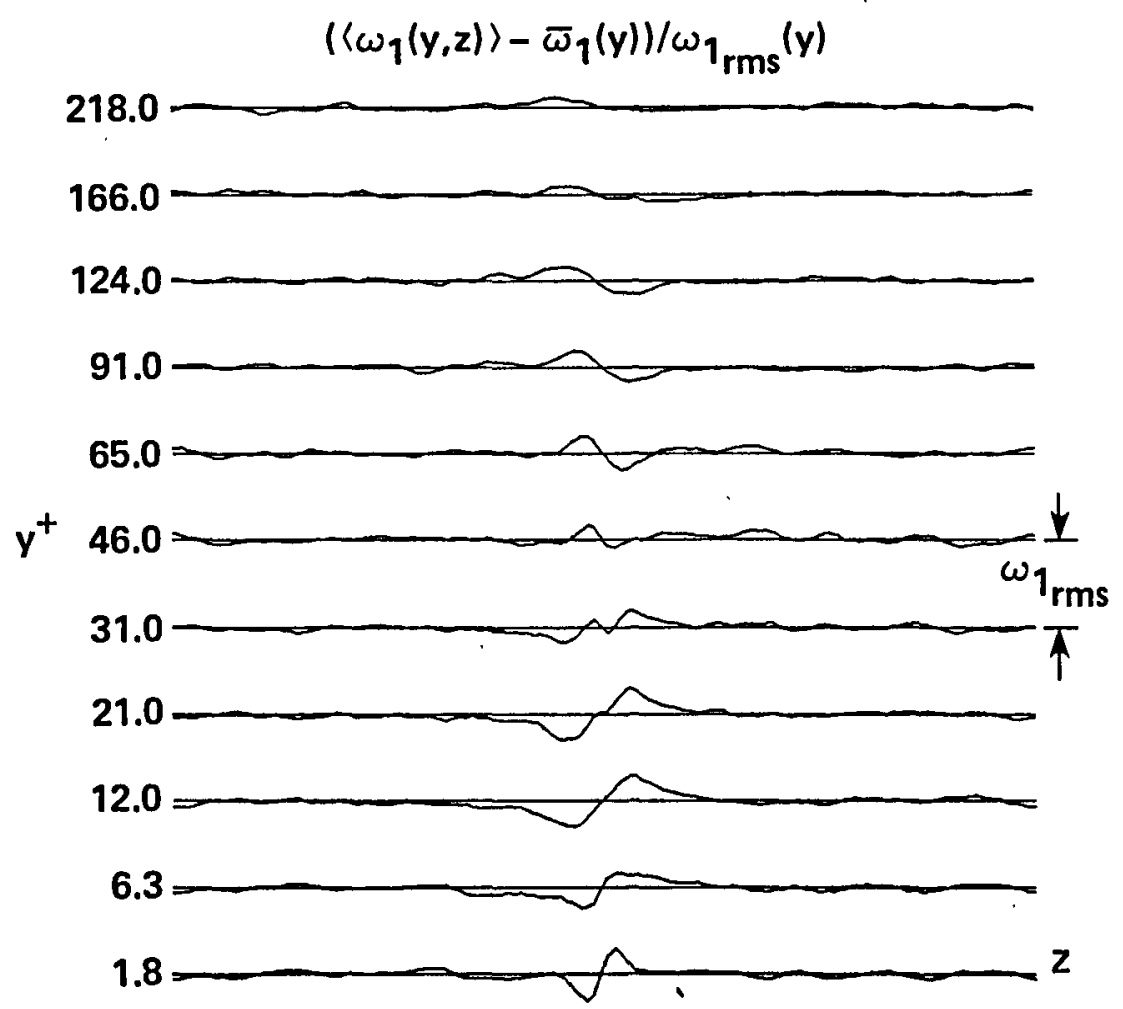

Fig. $8 f$ 


$$
\left.\mid\left\langle\omega_{1}(y, z)\right\rangle-\bar{\omega}_{1}(y)\right) / \omega_{1_{\mathrm{rms}}}(y)
$$

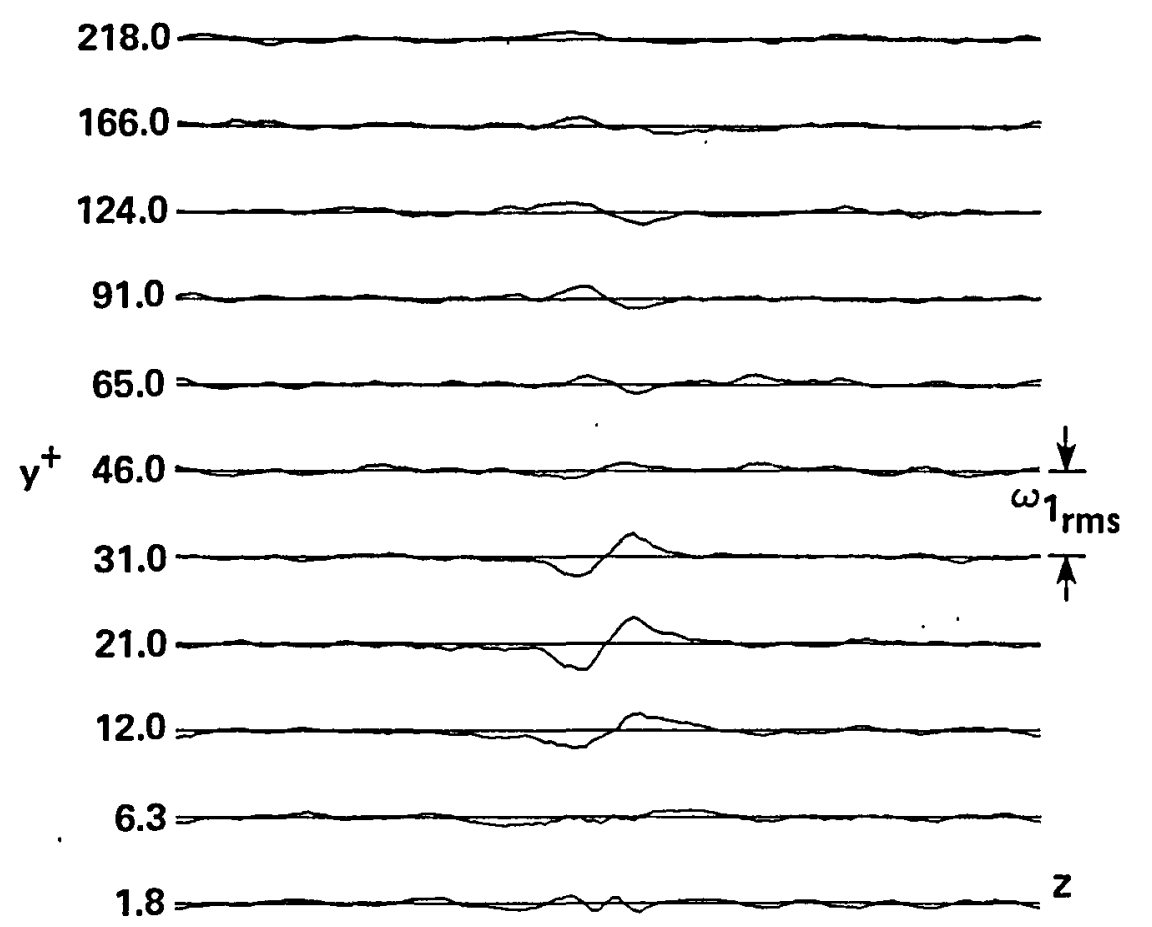

Fig. $8 g$ 


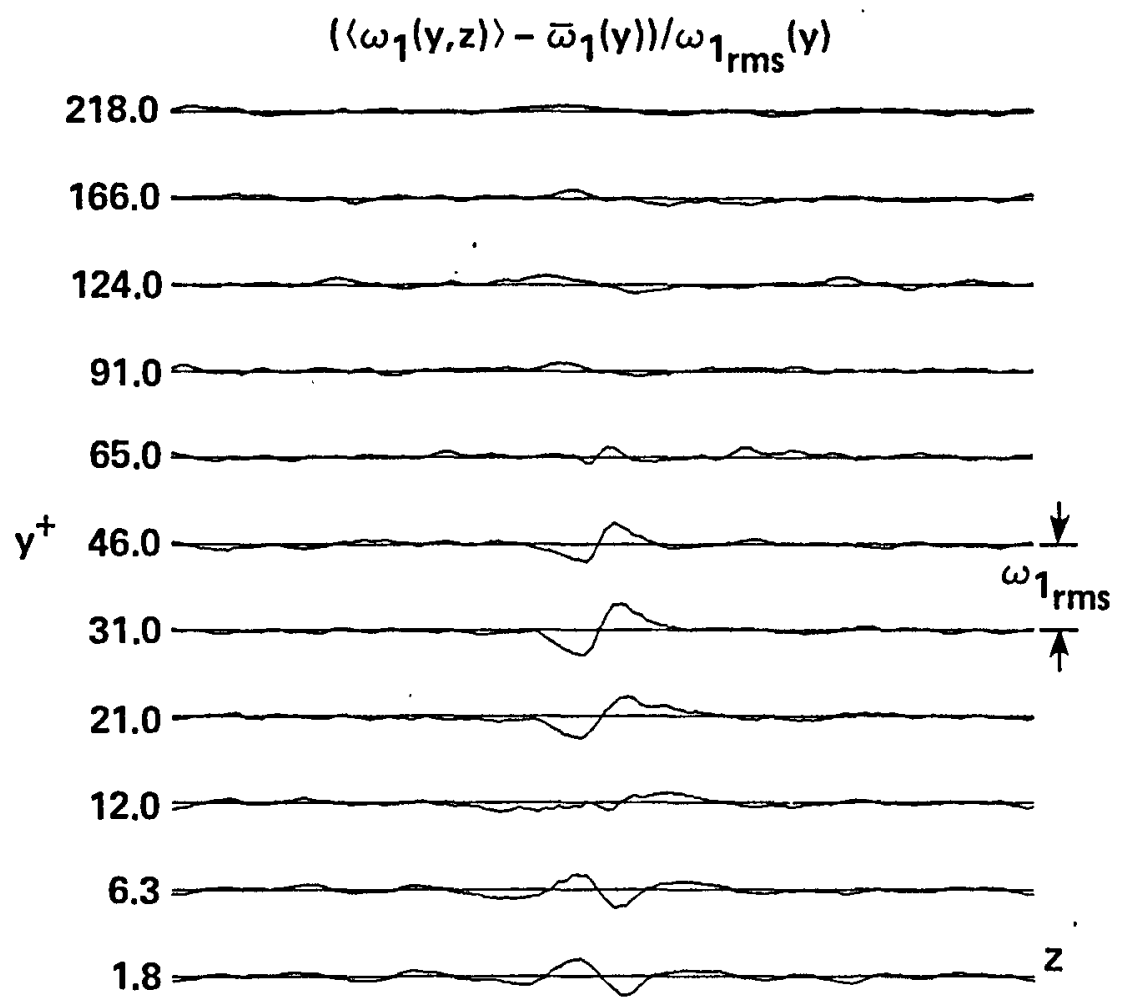

Fig. $8 \mathrm{~h}$ 


$$
\left.\mid\left\langle\omega_{1}(y, z)\right\rangle-\bar{\omega}_{1}(y)\right) / \omega_{1_{\mathrm{rms}}}(y)
$$

\section{0}

166.0

124.0

91.0

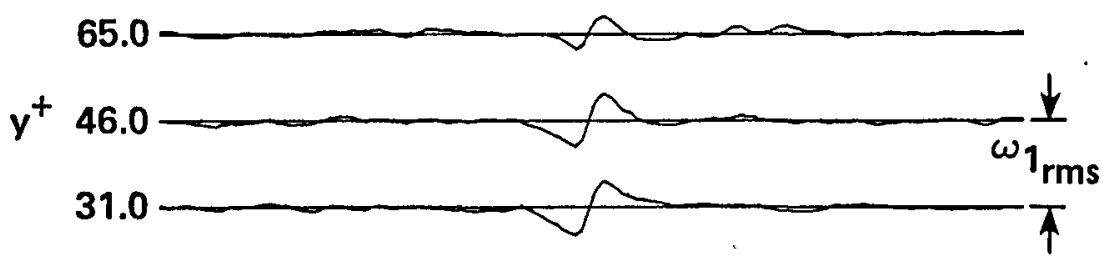

21.0

12.0

6.3

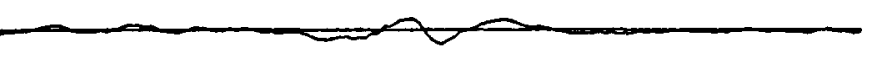

1.8
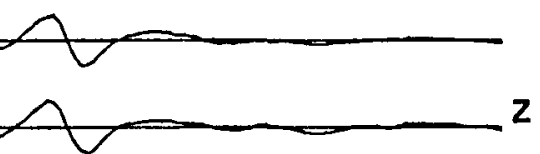

Fig. $3 i$ 

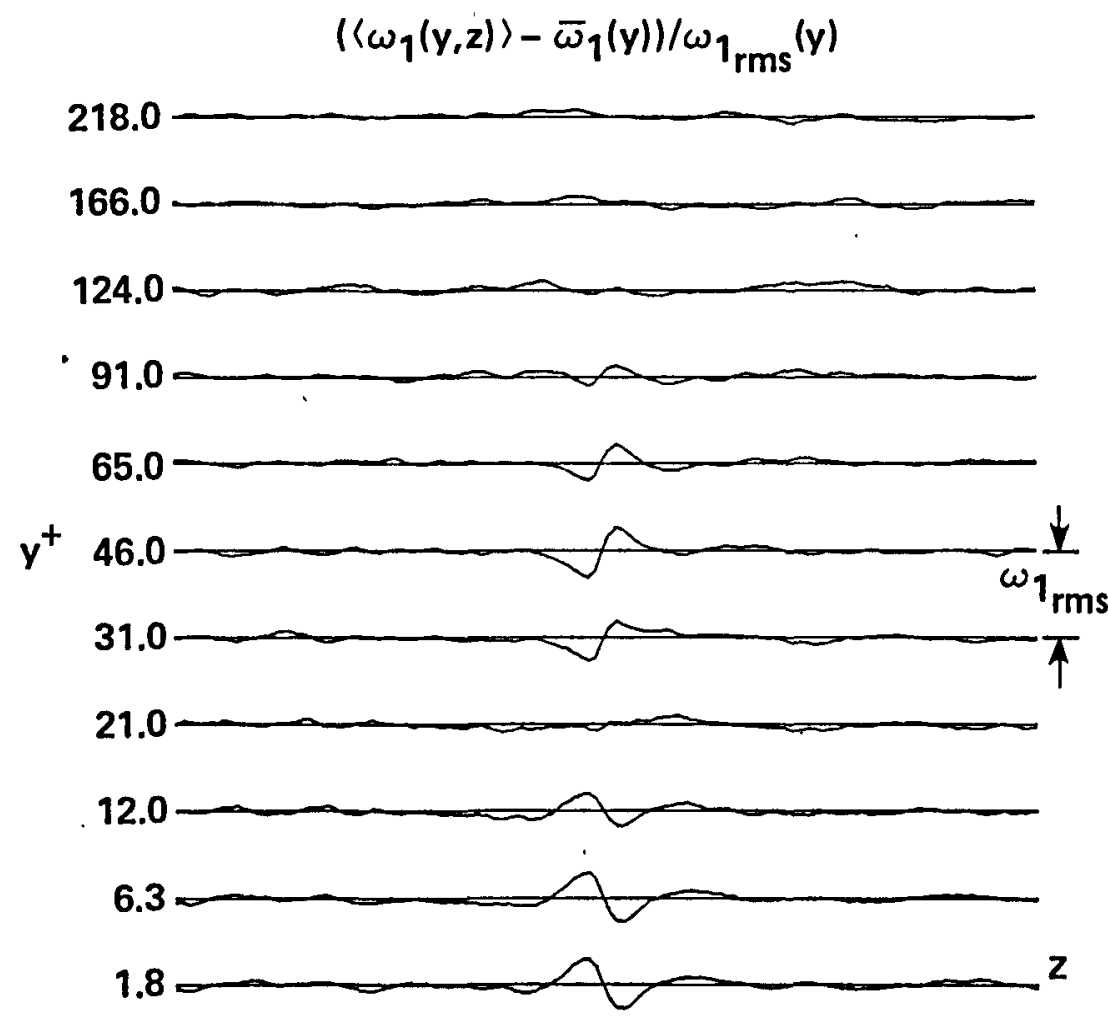

Fig. $8 j$ 

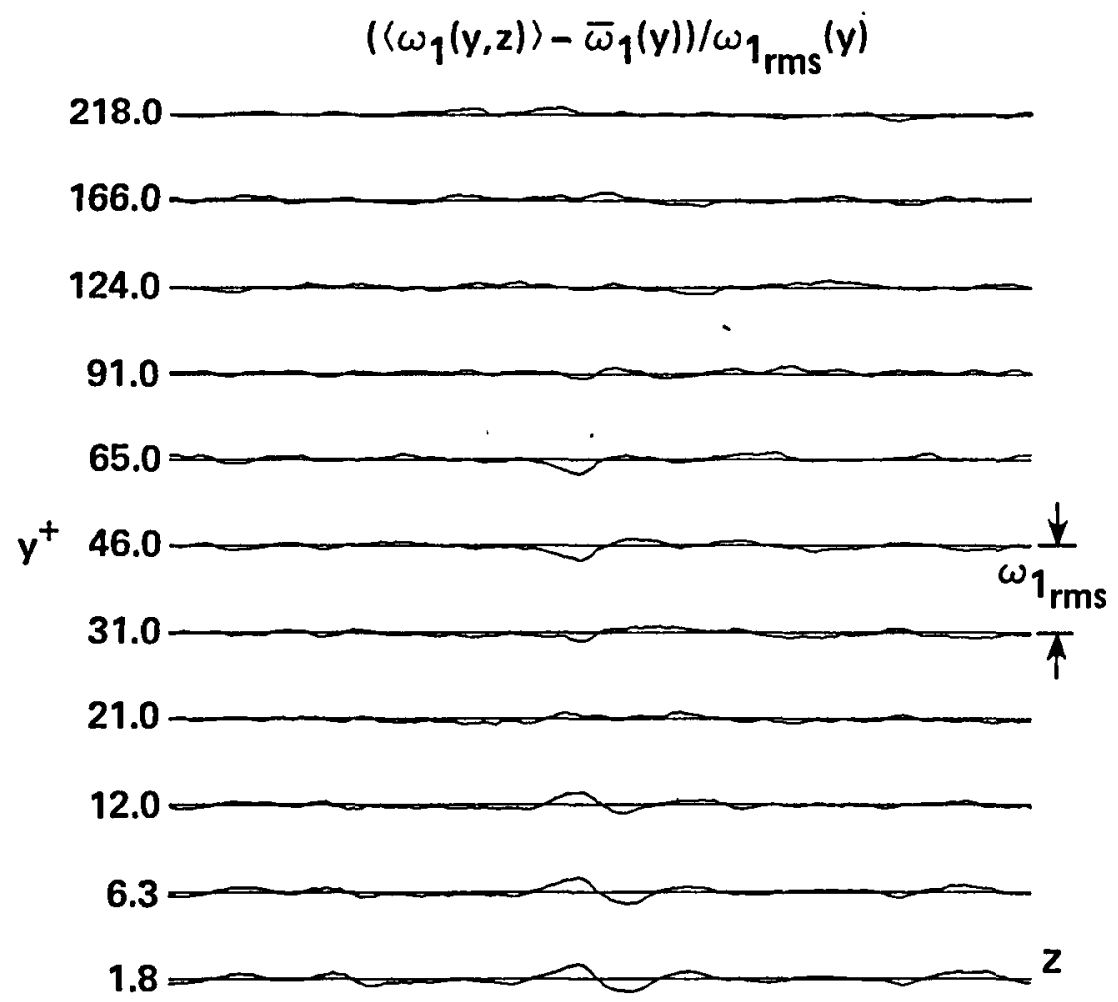

Fig. $8 k$ 

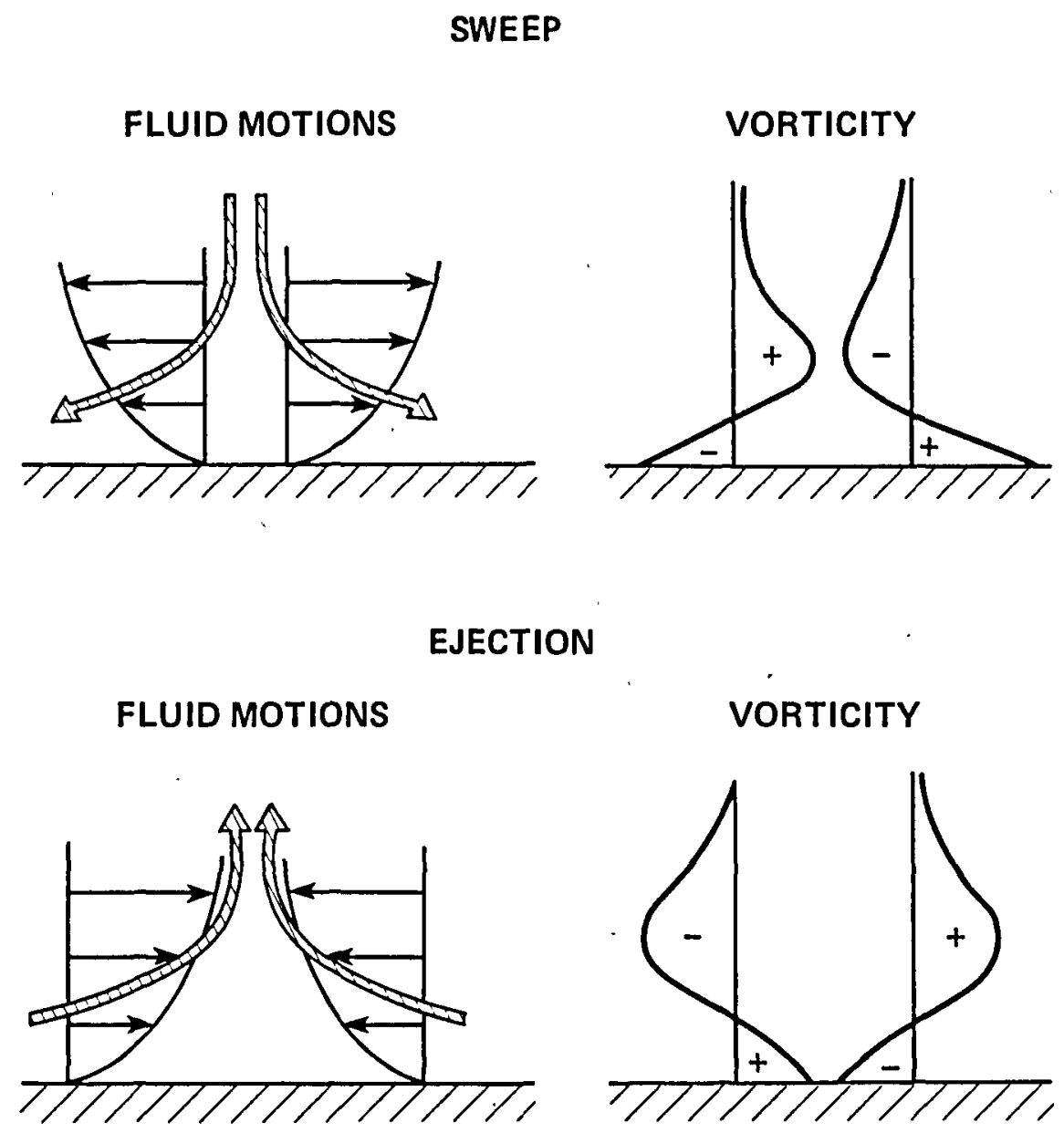

Fig. 9a 


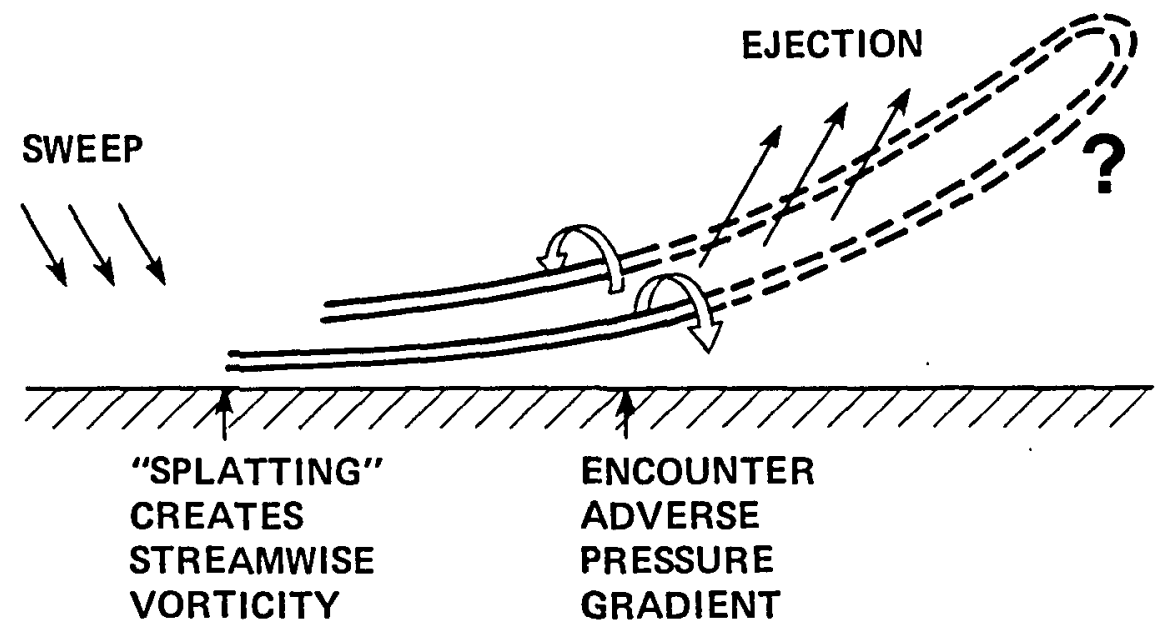

Fig. 9b 


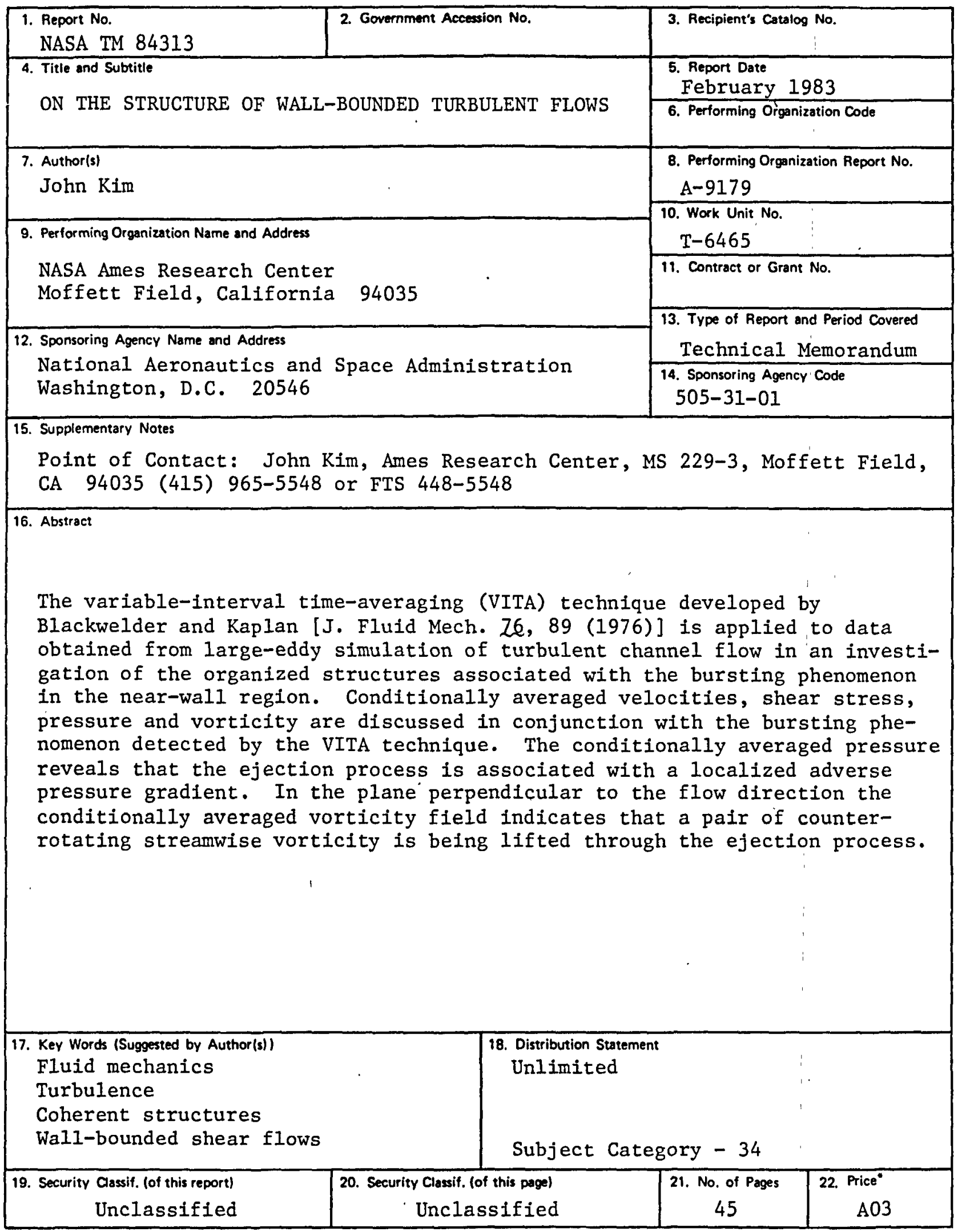

-For sale by the National Technical Information Service, Springfield, Virginia 22161 